\title{
A!
}

This is an electronic reprint of the original article.

This reprint may differ from the original in pagination and typographic detail.

G., David Gonzalez; Hakula, Harri; Rasila, Antti; Hamalainen, Jyri

\section{Spatial Mappings for Planning and Optimization of Cellular Networks}

Published in:

IEEE-ACM TRANSACTIONS ON NETWORKING

DOI:

10.1109/TNET.2017.2768561

Published: 01/01/2018

Document Version

Publisher's PDF, also known as Version of record

Please cite the original version:

G., D. G., Hakula, H., Rasila, A., \& Hamalainen, J. (2018). Spatial Mappings for Planning and Optimization of Cellular Networks. IEEE-ACM TRANSACTIONS ON NETWORKING, 26(1), 175-188.

https://doi.org/10.1109/TNET.2017.2768561

This material is protected by copyright and other intellectual property rights, and duplication or sale of all or part of any of the repository collections is not permitted, except that material may be duplicated by you for your research use or educational purposes in electronic or print form. You must obtain permission for any other use. Electronic or print copies may not be offered, whether for sale or otherwise to anyone who is not an authorised user. 


\title{
Spatial Mappings for Planning and Optimization of Cellular Networks
}

\author{
David González G. ${ }^{\circledR}$, Member, IEEE, Harri Hakula, Antti Rasila ${ }^{\circledR}$, and Jyri Hämäläinen, Member, IEEE
}

\begin{abstract}
In cellular networks, users are grouped into different cells and served by different access points (base stations) that provide wireless access to services and applications. In general, the service demand is very heterogeneous, non-uniformly distributed, and dynamic. Consequently, radio access networks create very irregular topologies with more access points, where service demand is concentrated. While this dynamism requires networks with the ability to adapt to time-varying conditions, the non-uniformity of the service demand makes the planning, analysis, and optimization difficult. In order to help with these tasks, a framework based on canonical domains and spatial mappings (e.g., conformal mapping) have recently been proposed. The idea is to carry out part of the planning in a canonical (perfectly symmetric) domain that is connected to the physical one (real-scenario) by means of a spatial transformation designed to map the access points consistently with the service demand. This paper continues the research in that direction by introducing additional tools and possibilities to that framework, namely the use of centroidal Voronoi algorithms and non-conformal composite mappings. Moreover, power optimization is also introduced to the framework. The results show the usability and effectiveness of the proposed method and its promising research perspectives.
\end{abstract}

Index Terms - Cellular networks, network planning, conformal mapping, power optimization, Voronoi tessellations.

\section{INTRODUCTION}

\section{A. Context and Motivation}

$\mathbf{R}$ ADIO access planning and optimization are fundamental tasks in cellular networks. Broadly speaking, planning refers to the tasks of determining the number, location, and configuration of access points to provide wireless access to users (and things) to services and applications, with a certain targeted Quality of Service (QoS). In particular, the problem of finding the number of access points is also referred to as dimensioning [1], and this initial step aims at providing the required capacity for the service demand volume that is expected. However, in practice, both dimensioning and sites positioning are very difficult problems because the service demand is not uniformly distributed and it is quite diverse and dynamic. Nowadays, taking into account the continuous evolution of radio access technologies, and the new concepts

Manuscript received March 21, 2017; revised September 19, 2017; accepted October 26, 2017; approved by IEEE/ACM TRANSACTIONS ON Networking Editor X. Liu. Date of publication November 15, 2017; date of current version February 14, 2018. This work supported by the Academy of Finland under Grant 287249, Grant 284811, and Grant 284634. (Corresponding author: David González G.)

D. González G. and J. Hämäläinen are with the Department of Communications and Networking, Aalto University, 02150 Espoo, Finland (e-mail: david.gonzalez.g@ieee.org).

H. Hakula and A. Rasila are with the Department of Mathematics and Systems Analysis, Aalto University, 02150 Espoo, Finland.

Digital Object Identifier 10.1109/TNET.2017.2768561 and paradigms that are expected for the fifth generation $(5 \mathrm{G})$ of cellular networks, the boundary between planning and optimization tasks becomes blurred. Indeed, according to the excellent work presented in [1], planning and optimization are iterative tasks that go hand-in-hand. In this line of thinking, Wang and Ran [2] also pose the need for re-thinking planning. They emphasize the importance of distributing the service demand as evenly as possible among cells as a key criterion to achieve effective planning; a goal that in the opinion of Wang and Ran [2] (and in our's) is a very valid way to enhance system performance. Planning of small cells and energy efficient operation of cellular infrastructure are indeed very active research topics. Interested readers are referred to [3] and [4], and the references therein, for an updated view and treatment of the matter.

In our previous work [5], also motivated by the aforementioned ideas, a novel framework for planning and optimization based on the use of canonical domains and spatial transformations was presented. Therein, planning is addressed by breaking the problem into two parts: dimensioning and sites positioning, as it is shown in Fig. 1. The central idea is to carry out the dimensioning in a dual/canonical domain in which the service demand is uniformly distributed. In this manner, a regular network topology $\left(\mathcal{T}_{c}\right)$ with the required number of access points is obtained (Step 2). Then, sites positioning is performed by mapping the regular network topology $\left(\mathcal{T}_{\mathrm{c}}\right)$ from the canonical domain $(\mathcal{R})$ onto the physical domain $(\mathcal{A})$ by means of a spatial mapping $F^{-1}: \mathcal{R} \rightarrow \mathcal{A}$ that corresponds to the inverse of another (previously computed) mapping $F: \mathcal{A} \rightarrow \mathcal{R}$. The idea is that, if the mapping $F$ is designed (Step 1) such that it maps $\mathcal{A}$ onto $\mathcal{R}$ redistributing the service demand uniformly, then $F^{-1}$ will map (Step 3) the access points from $\mathcal{R}$ onto $\mathcal{A}$ in a compatible manner with the service demand, i.e., the network topology $\mathcal{T}_{\mathrm{p}}$. In [5], conformal mapping [6], a mature field in Complex Analysis [7], but previously unheard of in the context of cellular networks, was proposed to obtain the mappings $F$ and $F^{-1}$.

The previous idea was a completely new approach to radio access network planning. However, as a new methodology, the scheme presented in [5] has two fundamental limitations: first, it cannot be used to address arbitrary non-uniform service demand distributions, and second, it lacks of means to adjust the transmit power of the access points. As it will be seen shortly, these are critical (required) enhancements to continue the evolution of this idea, as it has happened with other important techniques. We think that the development of this idea can be compared with the early days of Multiple-Input Multiple-Output (MIMO). First, well-acknowledged papers at 90's (e.g., paper by Foschini in 1996 [8]) introduced the idea of transmitting different data streams from different antennas and some simple reception methods (e.g., VBLAST).

1063-6692 @ 2017 IEEE. Translations and content mining are permitted for academic research only. Personal use is also permitted,

but republication/redistribution requires IEEE permission. See http://www.ieee.org/publications_standards/publications/rights/index.html for more information. 


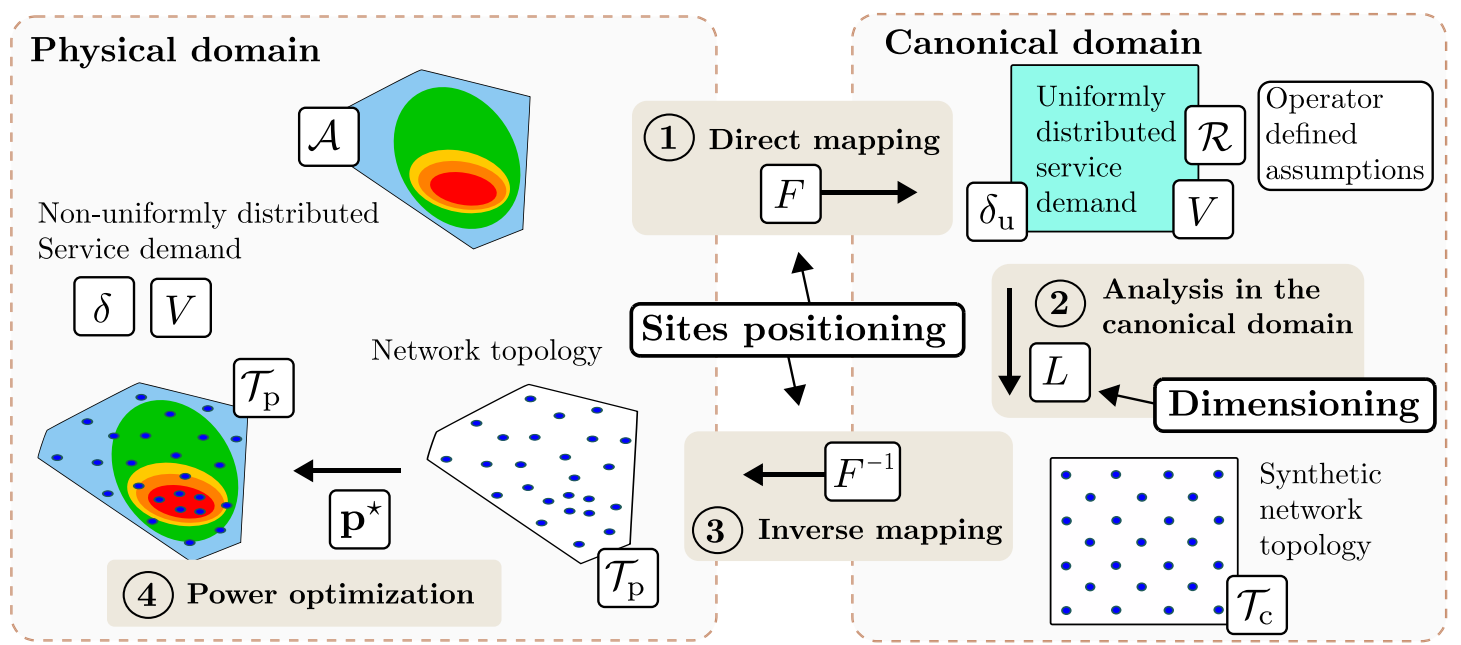

Fig. 1. Planning and optimization based on canonical domains and spatial mappings. The original problem introduced in [5] comprised Steps 1,2 , and 3 (direct and inverse mapping and analysis in the canonical domain). The proposed enhanced framework includes: generalizations for Step 1, alternative methods for Step 3, and integration of Step 4 (power optimization).

Thereafter, especially the signal processing community has made substantial efforts to further develop MIMO, but it has taken until $3.5 \mathrm{G}$ and $4 \mathrm{G}$ when simple MIMO schemes have been realized in commercial products. Network planning is an integral part of the development of every mobile network. Therefore, the authors believe that the idea proposed in [5] and extended in this paper has great potential. Yet, as MIMO in its early days, this concept needs a lot of methodological developments before it can become a (practically viable) mainstream approach. Part of the challenge is that some parts of the mathematical framework (e.g., conformal mapping) are not very well acknowledged within the signal processing and communications communities, and only few researchers have basic skills that would support research on this topic.

Finally, let us shortly discuss about the nature of the contribution to make clear the main goal of this research and its scope. We emphasize that our goal has not been to create a technical methodology or algorithm that aims at competing with existing network planning methods. There are many effective heuristic network planning methods applying e.g. genetic algorithms, and commercial tools that are routinely used to create practically viable network deployments. Instead, our main goal has been to develop a deterministic methodology that provides a new general theoretical framework that can be used to analyze and shed more light on the dependencies between different factors (service demand, load, channels, etc). We also note that in the applied approach, spatial mappings carry the information about the cellular topology. In system analysis this means that a rich mathematical machinery on mappings becomes a viable tool while studying the properties of the topology. Of course, it is also our goal that the algorithmic realization of the proposed methodology becomes in long run competitive with mainstream heuristic algorithms, but at this stage of research and development, synthetic scenarios, capturing the irregular features of real-world deployments, suffice as proof of concept.

\section{B. Contribution}

As mentioned before, the idea introduced in [5] is new and it represents a novel approach to network planning and optimization. However, as a technique in its infancy, it admits (and requires) further improvements and evolution. Hence, we continue the work on this research problem 1) by enhancing the capabilities of the originally introduced scheme with new concepts and tools (Steps 1 and 3 in Fig. 1), and 2) by adding new optimization mechanisms (Step 4). To be precise, the contributions of this paper can be summarized as follows:

$\checkmark$ The direct mapping (Step 1 in Fig. 1) has been generalized by introducing the notion of function composition [9]. With this novelty, it is possible to deal with any arbitrary spatial service demand distribution defined in the physical domain, a clear limitation of the original scheme. Thus, the new direct mapping includes both conformal and non-conformal transformations.

$\checkmark$ A new method for computing the inverse mapping (Step 3 in Fig. 1) using the centroidal Voronoi algorithms [10] and power Voronoi diagrams [11] has been introduced. As it will be explained later on, this approach does not require the computation of the direct mapping $F$. This is another substantial enhancement because, as it will be shown and explained later on, depending on the scale of the problem and some other features, one or other approach is preferred for the inverse mapping. In addition, this also provides means for comparisons, thus improving our understanding of the problem.

$\checkmark$ Power optimization, the new Step 4 in Fig. 1, has been introduced. This was motivated by the need for adjusting the transmit power of the access points once they are mapped onto the physical domain. The optimization formulation (more precisely, the proposed objective function) is novel, and it is based in the well-accepted model of load coupling [12] to represent in a quite realistic manner the intercell interference and the crosseffect caused by the load at different cells. As it will be seen, the proposed formulation has several interesting advantages from planning and network operation point of view.

The rest of the paper is organized as follows: the next section provides a high level description of the enhanced framework. Generalizations and novelties for the spatial mappings are presented in Sections III and V. The analysis 
TABLE I

NOTATIONS

\begin{tabular}{rlrl}
\hline Symbol & Description & Symbol & Description \\
\hline $\mathcal{A}$ & Physical domain & $\mathcal{R}$ & Canonical domain \\
$\mathcal{T}_{\mathrm{p}}$ & Topology in the physical domain & $\mathcal{T}_{\mathrm{c}}$ & Topology in the canonical domain \\
$\delta$ & Spatial service demand distribution & $\delta_{\mathrm{u}}$ & Uniform service demand distribution \\
$L$ & Number of base stations & $\mathbf{p} \in \mathbb{R}_{+}^{L}$ & Power allocation (in data channels) \\
$V$ & Service demand volume & $V_{l}$ & Service demand in the $l^{\text {th }}$ cell \\
$F$ & Direct mapping & $F^{-1}$ & Inverse mapping \\
$\mathbf{p}^{\star} \in \mathbb{R}_{+}^{L}$ & Optimized power allocation & $B$ & System bandwidth \\
$R_{\text {min }}$ & Minimum user rate & $\beta$ & Propagation exponent \\
$\bar{\alpha}_{\mathrm{c}}$ & Target cell load in $\mathcal{R}$ & $\bar{\alpha}$ & Resulting uniform cell load in $\mathcal{R}^{\prime}$ \\
\hline
\end{tabular}

in the canonical domain is, for the sake of completeness, briefly described in Section IV; it can be done following the methodology presented in [5, Sec. IV]. The proposed power optimization is described in Section VI. Numerical examples are presented in Section VII. Section VIII closes the paper with conclusions and future research directions.

\section{FRAMEWORK DESCRIPTION AND SYSTEM MODEL}

In general terms, planning aims at determining the number and location of base stations and their corresponding cell areas. The research framework under consideration, shown in Fig. 1, is motivated by the notion of service demand and capacity provision compatibility [13], in which more access points (with smaller cell areas) are required where the service demand is concentrated. Thus, if base stations have the same amount of resources, the ideal system should be planned and configured so that cells are equally loaded. However, determining such network topology and configuration is not an easy task because, in practice, the service demand is nonuniformly distributed in the coverage area.

In the proposed framework, two domains are considered. A physical domain $\mathcal{A}$ that corresponds to the real-world, and a dual canonical domain represented by a rectangular area $\mathcal{R}$. The service demand in the physical domain is assumed to be known in statistical terms, i.e., its spatial distribution given by a probability density function $\delta$ defined over $\mathcal{A}$, such that $\int_{\mathcal{A}} \delta(a) d a=1$, and a certain volume $V$, expressed in terms of the average number of users, are known. Evidently, both $\delta$ and $V$ vary over time, but for planning purposes, it can be fairly assumed that a given $\delta$ and $V$ (well-known by operators) capture the traffic behavior in representative periods of time [14]-[16], i.e., morning, peak-hour, afternoon, and so on.

As in [5], the target is to find a network topology $\mathcal{T}$ (site's locations and cell areas) with a certain configuration (e.g., power allocation) that is compatible with the service demand and satisfies both coverage and capacity requirements. The proposed framework is composed of the four main steps indicated in Fig. 1 and explained next:

1) Direct Mapping: The objective is to determine a mapping function $F: \mathcal{A} \rightarrow \mathcal{R}$, such that the service demand that is non-uniformly distributed (according to $\delta$ ) in $\mathcal{A}$ becomes uniform in $\mathcal{R}$. The uniform service demand distribution in $\mathcal{R}$ is denoted by $\delta_{\mathrm{u}}$, and hence, $\delta_{\mathrm{u}}(r)=\frac{1}{|\mathcal{R}|}, \forall r \in \mathcal{R}$. Therefore, the function $F$ must be a function of $\delta$. The novel direct mapping proposed herein (Step 1), based on composition of conformal and non-conformal mapping, is discussed in Section III.
2) Analysis in the Canonical Domain: The goal is to determine the number $L$ of uniformly distributed access points required to satisfy the uniformly distributed service demand in $\mathcal{R}$. Hence, $L$ depends on $V$, i.e., the larger the volume, the higher the density of the uniform topology $\mathcal{T}_{\mathrm{u}}$. Note that the same volume $V$ is considered in both domains. A description of the analysis in the canonical domain (Step 2) is provided in Section IV.

3) Inverse Mapping: The target is to find a spatial transformation $F^{-1}: \mathcal{R} \rightarrow \mathcal{A}$ to map the access points from the canonical domain onto the physical domain. If $F$ has been defined, $F^{-1}$ corresponds to its inverse function, and hence, it also depends on $\delta$. However, one of the novelties introduced herein allows to obtain the mapping $F^{-1}$ directly from $\delta$, i.e., without need for inverting $F$. Thus, new possibilities are available. In any case, the important point is that the inverse mapping conveys the information stored in $\delta$ (the spatial service demand distribution). Thus, the mapping $F^{-1}$ guarantees that the resulting network topology $\mathcal{T}$ in the physical domain is spatially compatible with the service demand, and on the other hand, the $L$ access points, previously calculated for $\mathcal{T}_{\mathrm{u}}$, provide the required capacity to deal with the service demand volume. The aforementioned alternatives for the computation of the inverse mapping (Step 3) are presented in Section V.

4) Power Optimization: Depending on the amount of nonuniformity of $\delta$ (how much traffic is concentrated in the areas with high demand) and the network density (proportional to $L$ ), power optimization is required to compensate the high interference that would be generated in the hot spots. As indicated, regions with higher demand require smaller cells, and hence, the transmit power needs to be adjusted to avoid unfeasible interference levels, and to equalize the load of the different cells. The proposed power optimization (Step 4) aims at equalizing load levels network-wise. It is presented in Section VI.

In order to facilitate the reading of the rest of the article, the notation is presented in Table I.

\section{DiRECT MAPPING}

The objective of the direct mapping $F$ is to uniformly distribute the service demand from the physical domain onto the canonical domain. To do that, the mapping is required to stretch or compress the space according to $\delta$, i.e., if $\delta$ admits high values in some region of $\mathcal{A}$, then that region needs to be mapped onto a larger image in $\mathcal{R}$, and viceversa. This idea 


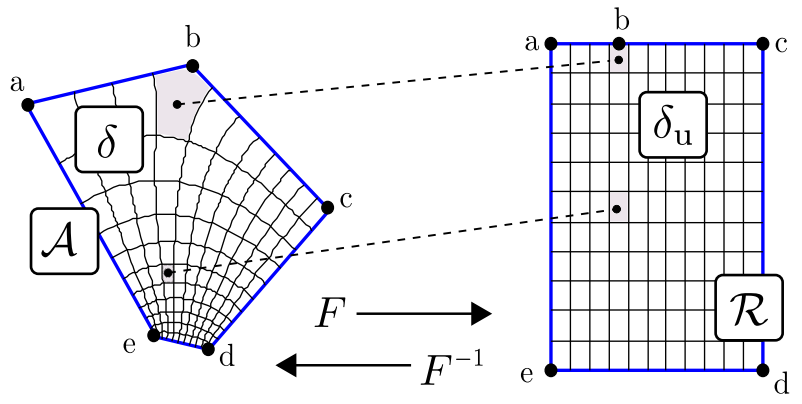

(a)

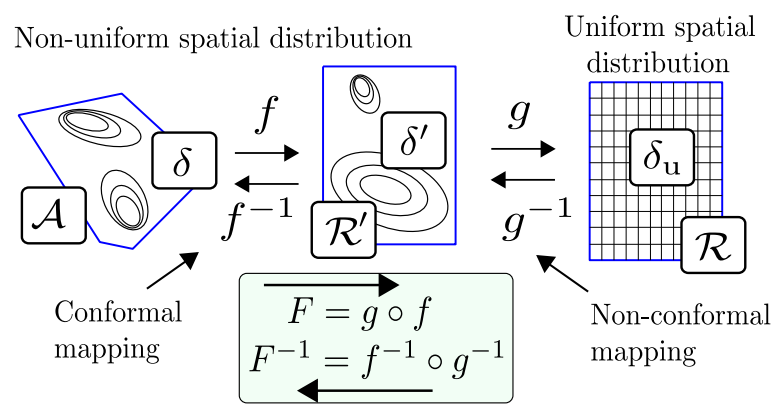

(b)

Fig. 2. The direct mapping re-distributes the service demand uniformly in the canonical domain. (a) Space deformation produced by the mapping. (b) Mapping composition: polygon $\leftrightarrow$ rectangle.

is illustrated in Fig. 2a, where the spatial service demand distribution is represented by a grid in both domains. Each subregion of the grid has the same amount of traffic. Note that in $\mathcal{R}$, the grid is uniform, and hence, service demand $\delta_{\mathrm{u}}$ is also uniform, while in $\mathcal{A}$ is not, i.e., the traffic is concentrated where the grid is denser. In the example, two regions of each domain are connected through the mappings $\left(F\right.$ and $\left.F^{-1}\right)$, therefore, they are images of each other. It can be seen that, the regions with high traffic density in $\mathcal{A}$ are mapped to larger regions in $\mathcal{R}$, and viceversa. Thus, the mappings not only connect (bijectively) both domains preserving notions such as locality and proximity, but also carries information about the service demand distribution. In [5], the analysis through canonical domains was presented by implementing the direct mapping $F$ by means of conformal mapping [17], to be precise, a mapping from polygon onto rectangle using modified Schwarz-Christoffel transformations [18]. In order to proof the concept, $\delta$ was assumed to be proportional to the natural deformation of the space produced by the conformal mapping used to connect $\mathcal{A}$ and $\mathcal{R}$. However, despite a certain flexibility to model $\delta$ when defining the mapping (by conveniently modifying the boundaries and vertices in $\mathcal{A}$, and selecting the corners of the generalized cuadrilateral [6]), the method has limitations. Thus, an improved solution is provided herein to address the more general case when an arbitrary $\delta$ is defined in the physical domain. Given that, to the best of the knowledge of the authors, there are not known methods to conformally map polygons onto rectangles and at the same time redistribute uniformly an arbitrary spatial service demand distribution defined on it; the solution presented herein essentially divides the problem into two pieces as shown in Fig. 2b. The idea is to construct the mapping $F: \mathcal{A} \rightarrow \mathcal{R}$ as a composition of two mappings: an initial conformal mapping $f: \mathcal{A} \rightarrow \mathcal{R}^{\prime}$, and a second one $g: \mathcal{R}^{\prime} \rightarrow \mathcal{R}$, such that $F=g \circ f$. The first mapping focuses on the problem of mapping the physical domain (a given polygon) onto the canonical domain (a rectangle), for which complex analysis, i.e., conformal mapping, is required. In general, an arbitrary spatial service demand distribution $\delta$ defined over $\mathcal{A}$ will be transformed into another non-uniform distribution $\delta^{\prime}$ defined over $\mathcal{R}^{\prime}$. Therefore, a second mapping $g$ is required to homogenize $\delta^{\prime}$. However, this second mapping has a fundamental difference with respect to the first one: it is a mapping between rectangular domains (indeed, $\mathcal{R}$ and $\mathcal{R}^{\prime}$ can be identical), and hence, complex analysis is not longer required.

The mapping $f$ is identical to the one described in [5, Sec. III-B], that was originally proposed in [18].
Details on the computation of $f$ and its inverse $f^{-1}$ can be found therein. Additional useful information on conformal mapping, Schwarz-Christoffel transformations, and equivalence among quadrilaterals can be found in [6] and [19].

Focusing on the non-conformal mapping ( $g$ and $g^{-1}$, see Fig. 2b), it is important to indicate that the main reason for calculating the direct mapping $g$ is to obtain its inverse $g^{-1}$. Recall that in the proposed framework, the inverse mapping is the one used to create the network topology $\mathcal{T}_{\mathrm{p}}$ in the physical domain (sites positioning) once the analysis in the canonical domain is completed. However, if $g^{-1}$ can be obtained directly by means of $\delta^{\prime}$, then $g$ can also be computed by finding the inverse of $g^{-1}$, but in this case it is not strictly necessary. This is the approach used herein, and hence, the computation of $g^{-1}$ is presented in Section V.

It should be noticed that since the network topology created in $\mathcal{R}^{\prime}$ and the spatial distribution $\delta^{\prime}$ are both non-uniform, $\mathcal{R}^{\prime}$ can be regarded as an intermediate physical domain in which the use of the power optimization proposed in Section VI can be illustrated without loss of generality.

\section{AnAlysis in the CANONICAL Domain}

The analysis in the canonical domain addresses the dimensioning part of the planning problem, i.e., to determine how many access points $L$ are required to cope with the service demand, and more precisely with the given volume $V$. A full description of the method can be found in [5, Sec IV]. Nevertheless, a brief description is also provided herein for the sake of completeness. One of the key objectives in [5] is to perform and simplify part of the planning process by carrying out part of the job in a dual domain, referred to as canonical domain, that is 1) perfectly regular in terms of the geometry of the network topology, 2) uniform in terms of spatial service demand distribution, and 3) homogeneous in terms of interference. In this manner, independently of the density (proportional to $L$ ) that is considered, cells are identical in terms of coverage area, service demand, and interference, hence, the analysis of one single cell suffices. In order to do that, a rectangular domain was selected because it is topologically equivalent to the flat torus, a 2D manifold [20], in which the condition of periodicity can be applied to avoid border effects. Thus, cells become equally loaded and the dimensioning is conservative since the wrap-around produced by the periodicity implies the worst case in terms of interference. The analysis presented in [5] takes into account the cell load coupling model originally presented in [12], but simpler assumptions such as full load can also be considered. 


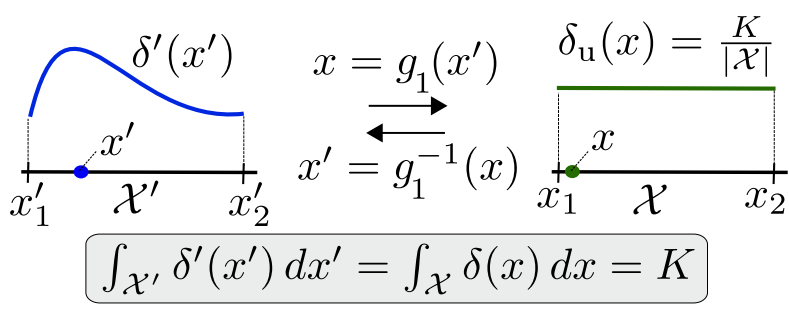

(a)

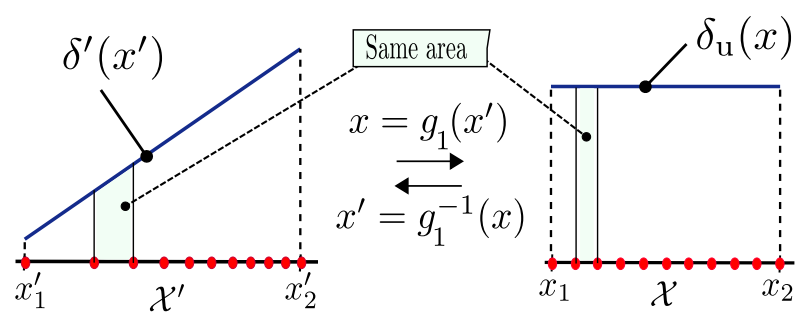

(b)

Fig. 3. Required service demand redistribution (mapping) in one dimension. (a) Notation and baseline assumptions. (b) Mapping in $1 \mathrm{D}$ with $\delta^{\prime}\left(x^{\prime}\right)$ as a linear function.

\section{INVERSE MAPPING}

In this work, two alternatives for finding $F^{-1}$ are presented. The first option is to express $F^{-1}$ in terms of a composition of two functions as indicated in Fig. 2b, i.e., $F^{-1}=f^{-1} \circ g^{-1}$. As mentioned before, the computation of the conformal mapping $f^{-1}$ is described in [5] and [18], and the references therein. Hence, with this approach, the task is reduced to obtain $g^{-1}$. The second alternative is to use an algorithmic solution to estimate $F^{-1}$. In this work, this approach is based on Centroidal Voronoi Tessellations [10]. The idea and method was originally proposed for planning purposes in [21]. ${ }^{1}$ Both methods are described next.

\section{A. Non-Conformal Mapping}

In order to introduce the proposed mapping, let us to consider the mapping in one dimension $g_{1}: \mathcal{X}^{\prime} \rightarrow \mathcal{X}$ between two linear domains $\mathcal{X}$ and $\mathcal{X}^{\prime}$ as illustrated in Fig. 3a. The density function $\delta^{\prime} \in \mathbb{R}_{+}$is defined over $\mathcal{X}^{\prime}$ and the mapping $g_{1}$ is such that it uniformly re-distributes that demand in $\mathcal{X}$. Its inverse, $g_{1}^{-1}: \mathcal{X} \rightarrow \mathcal{X}^{\prime}$, on the other hand, must create $\delta^{\prime}$ when mapping a uniform density $\delta_{\mathrm{u}}$ from $\mathcal{X}$ onto $\mathcal{X}^{\prime}$. Note that, if $K=1, \delta^{\prime}$ and $\delta_{\mathrm{u}}$ in Fig. 3a can be regarded as probability density functions. Therefore, without loss of generality, hereafter $K$ is assumed to be equal to 1 . The mapping must fulfill the following conditions: 1) the total volume in each domain must be preserved, and hence, $\int_{\mathcal{X}^{\prime}} \delta^{\prime}\left(x^{\prime}\right) d x^{\prime}=\int_{\mathcal{X}} \delta_{\mathrm{u}}(x) d x=1$, and 2) $x_{1}=g_{1}\left(x_{1}^{\prime}\right)$ and $x_{2}=g_{1}\left(x_{2}^{\prime}\right)$. Intuitively, the function $g_{1}^{-1}$ has to map the points from $\mathcal{X}$ onto $\mathcal{X}^{\prime}$ in such a way that the area between two mapped points is the same in both domains as it is shown in Fig. 3b, where $\delta^{\prime}$ is assumed to be linear. Thus, the space is compressed in $\mathcal{X}^{\prime}$ if $\delta^{\prime}$ is high and streched is $\delta^{\prime}$ is low. In Fig. 3b, red points are images of each other to illustrate this idea. The required mapping $x^{\prime}=g_{1}^{-1}(x)$ can be obtained by solving for $x^{\prime}$ in the following expression:

$$
\int_{x_{1}^{\prime}}^{x^{\prime}} \delta^{\prime}\left(x^{\prime}\right) d x^{\prime}=\int_{x_{1}}^{x} \delta_{\mathrm{u}}(x) d x=\frac{x-x_{1}}{x_{2}-x_{1}} .
$$

Depending on $\delta^{\prime}, x^{\prime}=g_{1}^{-1}(x)$ can be expressed in closed form, but in general the mapping $g_{1}^{-1}$ can be evaluated numerically. Although the one-dimensional case is presented herein as a constitutive part of a the more general two-dimensional one, it can be used in practice in cases where the network deployment can be fairly represented in one dimension, e.g., road-side networks.

\footnotetext{
${ }^{1}$ The Short Paper format used in [21] only allowed to introduce the main idea and basic/preliminary results.
}

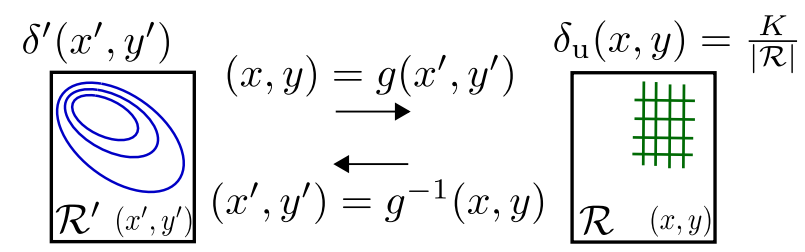

$$
\int_{\mathcal{R}^{\prime}} \delta^{\prime}\left(x^{\prime}, y^{\prime}\right) d x^{\prime} d y^{\prime}=\int_{\mathcal{R}} \delta_{\mathrm{u}}(x, y) d x d y=K
$$

Fig. 4. Required service demand redistribution (mapping) in two dimensions.

In two dimensions, the goal of the mapping is the same, i.e., to uniformly distribute a non-uniform service demand (volume) from a rectangular domain $\mathcal{R}^{\prime}$ onto another rectangular domain $\mathcal{R}$, as shown in the Fig. 4. In this case, there are two possibilities: 1) the spatial service demand distribution $\delta^{\prime}$ can be expressed as a product of two independent functions of $x^{\prime}$ and $y^{\prime}\left(\delta_{\mathrm{x}}^{\prime}\left(x^{\prime}\right)\right.$ and $\delta_{\mathrm{y}}^{\prime}\left(y^{\prime}\right)$, respectively), as follows: $\delta^{\prime}\left(x^{\prime}, y^{\prime}\right)=\delta_{\mathrm{x}}^{\prime}\left(x^{\prime}\right) \delta_{\mathrm{y}}^{\prime}\left(y^{\prime}\right)$, i.e., there is statistical independence between $x^{\prime}$ and $y^{\prime}$, and 2) $\delta^{\prime}\left(x^{\prime}, y^{\prime}\right)$ is given as a joint probability density function that cannot be expressed as a product of independent functions of $x^{\prime}$ and $y^{\prime}$. In the first case the mapping of each coordinate can be obtained independently by considering each dimension as the one-dimensional (linear) problem previously discussed, i.e., obtaining the mapping through (1). Thus, the mapping $g^{-1}$ can be written as follows:

$$
\left(x^{\prime}, y^{\prime}\right)=(u(x), v(y)),
$$

where $x^{\prime}$ only depends on $x$ and $y^{\prime}$ only depends on $y$.

If there is no statistical independence between the coordinates, there is no direct way to obtain the required mapping directly from $\delta^{\prime}\left(x^{\prime}, y^{\prime}\right)$. However, the solution for the onedimensional case, using (1), can be employed if the joint probability density function is marginalized [22]. In this case, the required mapping is not unique as it depends on the variable that is selected first, but in any case it fulfills the requirement of preserving the service demand volume between regions of both domains that are images of each other. The required mapping (assuming without loss of generality that the mapping for $x^{\prime}$ is taken first) can be obtained as follows:

1) Marginalize $\delta^{\prime}\left(x^{\prime}, y^{\prime}\right)$ with respect to $y^{\prime}$ to obtain the function $\delta_{\mathrm{x}}^{\prime}\left(x^{\prime}\right)$, which is given by

$$
\delta_{\mathrm{x}}^{\prime}\left(x^{\prime}\right)=\int \delta^{\prime}\left(x^{\prime}, y^{\prime}\right) d y^{\prime} .
$$

2) Obtain the mapping $x^{\prime}=u(x)$ by means of (1) and $\delta_{\mathrm{x}}^{\prime}\left(x^{\prime}\right)$. 
3) Use $\delta_{\mathrm{x}}^{\prime}\left(x^{\prime}\right)$ to build $\delta_{\mathrm{y}}^{\prime}\left(y^{\prime}, x\right)$ as follows:

$$
\delta_{\mathrm{y}}^{\prime}\left(y^{\prime}, x\right)=\frac{\delta^{\prime}\left(x^{\prime}, y^{\prime}\right)}{\delta_{\mathrm{x}}^{\prime}\left(x^{\prime}\right)}=\frac{\delta^{\prime}\left(u(x), y^{\prime}\right)}{\delta_{\mathrm{x}}^{\prime}(u(x))} .
$$

4) Obtain the mapping $y^{\prime}=v(x, y)$ by means of (1) and $\delta_{\mathrm{y}}^{\prime}\left(y^{\prime}, x\right)$.

Thus, the mapping $g^{-1}$ can be written as follows:

$$
\left(x^{\prime}, y^{\prime}\right)=(u(x), v(x, y)) .
$$

Analogously, and following the previous procedure, if $y^{\prime}$ is taken first, the mapping $g^{-1}$ would be given by

$$
\left(x^{\prime}, y^{\prime}\right)=(u(x, y), v(y)) \text {. }
$$

In both cases, in contrast to (2), one of mappings (either $u$ or $v$ ) is a function of two variables as indicated in (5) and (6).

\section{B. A Method Based on Centroidal Voronoi Tessellations}

A Voronoi diagram is a partition of a domain $\mathcal{A} \subset \mathbb{R}^{2}$ into $L$ regions $\left(\mathcal{A}_{l}, l=1,2, \cdots, L\right)$ that are associated to a subset of distinct points $\mathcal{L}=\left\{\boldsymbol{a}_{1}, \boldsymbol{a}_{2}, \cdots, \boldsymbol{a}_{L}\right\} \subset \mathcal{A} \subset \mathbb{R}^{2}$ where

$$
\mathcal{A}_{l} \triangleq\left\{\boldsymbol{a} \in \mathcal{A} \mid\left\|\boldsymbol{a}-\boldsymbol{a}_{l}\right\|_{2} \leq\left\|\boldsymbol{a}-\boldsymbol{a}_{k}\right\|_{2}, \forall l \neq k\right\} .
$$

The notation $\left\|\boldsymbol{a}_{1}-\boldsymbol{a}_{2}\right\|_{2}$ (the $L_{2}$-norm) indicates the Euclidean distance between the points $\boldsymbol{a}_{1}$ and $\boldsymbol{a}_{2}$. The points in $\mathcal{L}$ are known as generators of the Voronoi diagram. Voronoi diagrams have extensively been used in the analysis of cellular networks [23]-[25] because the definition of the Voronoi regions is consistent with the coverage areas (cells) of different Base Stations (BSs), i.e., under the assumption that BSs transmit pilots (used for cell selection [26]) with the same power, cells would correspond to regions defined by (7). If pilots are transmitted with different power levels, power Voronoi diagrams [11] can be used to determine cell regions. Power Voronoi diagrams can be regarded as a generalization of classic Voronoi diagrams by assigning weights to the generators (in this case BSs), thus locally defining the distance metric. The relation between the pilots' transmit power can be captured by the weights. In Power Voronoi diagrams, cell regions are defined as follows [11]:

$\mathcal{A}_{l} \triangleq\left\{\boldsymbol{a} \in \mathcal{A} \mid\left\|\boldsymbol{a}-\boldsymbol{a}_{l}\right\|_{2}-w_{l} \leq\left\|\boldsymbol{a}-\boldsymbol{a}_{k}\right\|_{2}-w_{k}, \forall l \neq k\right\}$.

A network topology $(\mathcal{T})$ in the domain $\mathcal{A}$ is defined as a set of BSs whose locations are indicated by the points $\mathcal{L}=\left\{\boldsymbol{a}_{1}, \boldsymbol{a}_{2}, \cdots, \boldsymbol{a}_{L}\right\} \subset \mathcal{A}$ and their corresponding cells $\left(\mathcal{A}_{l}\right.$ 's) are obtained by means of (8), with the weights $\mathcal{W}=\left\{w_{1}, w_{2}, \cdots, w_{L}\right\} \subset \mathbb{R}$. Thus, $\mathcal{T} \triangleq\left\{\mathcal{L}, \mathcal{A}_{l=1, \cdots, L}\right\}$.

The idea introduced in [21] is the joint use of Centroidal Voronoi Tessellations [10] and Power Voronoi diagrams [11] to obtain the topology $\mathcal{T}_{\mathrm{p}}$ (see Fig. 1) from the number of cells $L$ (obtained through the analysis in the canonical domain, Section IV) and $\delta$; thus achieving the goal of the mapping $F^{-1}$, i.e., to place the $L$ access points in $\mathcal{A}$ in a compatible manner with the spatial service demand distribution.

Before introducing the proposed heuristic, two notions are required: tessellation and mass centroid. A tessellation defined in a domain $\mathcal{A}$ corresponds to a set of regions $\mathcal{A}_{l} \subset \mathcal{A}, l=1, \cdots, L$, such that $\cup \mathcal{A}_{l}=\mathcal{A}$ and $\cap \mathcal{A}_{l}=\emptyset$. Thus, the sets defined by (7) and (8) correspond to tessellations in $\mathcal{A}$. The mass centroid $c_{l}$ of a region $\mathcal{A}_{l} \subset \mathcal{A}$ (evidently, $\left.\boldsymbol{c}_{l} \in \mathcal{A}_{l}\right)$ is defined as follows:

$$
\begin{aligned}
\boldsymbol{c}_{l} & \triangleq \frac{\int_{\mathcal{A}_{l}} \boldsymbol{a} \delta(\boldsymbol{a}) d \boldsymbol{a}}{\int_{\mathcal{A}_{l}} \delta(\boldsymbol{a}) d \boldsymbol{a}} \\
& \triangleq\left(\frac{\iint_{\mathcal{A}_{l}} x \delta(x, y) d x d y}{\iint_{\mathcal{A}_{l}} \delta(x, y) d x d y}, \frac{\iint_{\mathcal{A}_{l}} y \delta(x, y) d x d y}{\iint_{\mathcal{A}_{l}} \delta(x, y) d x d y}\right),
\end{aligned}
$$

where $\delta$ is a density defined over $\mathcal{A}$. In this context, $\delta$ corresponds to the spatial service demand distribution (see Fig. 1).

In centroidal Voronoi based algorithms, the idea is to start with an initial set of generators $\mathcal{L}^{\mathrm{R}}=\left\{\boldsymbol{a}_{1}^{\mathrm{R}}, \boldsymbol{a}_{2}^{\mathrm{R}}, \cdots, \boldsymbol{a}_{L}^{\mathrm{R}}\right\} \subset \mathcal{A}$, which can be selected randomly, and compute the corresponding Voronoi diagram and mass centroids $c_{l}$ of each cell $\mathcal{A}_{l}$ according to (9). Then, at each iteration, the $L$ centroids $\mathcal{C}^{i}=$ $\left\{\boldsymbol{c}_{0}^{i}, \boldsymbol{c}_{1}^{i}, \cdots, \boldsymbol{c}_{L}^{i}\right\}$ of the $i^{\text {th }}$ iteration are used as generators of the next Voronoi diagram, i.e., $\mathcal{L}^{i+1} \leftarrow \mathcal{C}^{i}$, till $\boldsymbol{a}_{l}^{i+1}=$ $c_{l}^{i}, \forall l$. The iterative mechanism is required because, in general, Voronoi generators and mass centroids do not match. Hence, the objective is to repeat this process till the generators of the Voronoi diagrams and mass centroids are the same [10].

If the spatial service demand is non-uniform, the centroidal Voronoi algorithm concentrates the access points where the demand is concentrated, i.e., where $\delta$ is high. Thus, the generators of the last Voronoi diagram (and their cells) define a network topology that is compatible with the service demand and that can be used for planning purposes. However, in [21] an improvement based on Power Voronoi diagrams was proposed to obtain network topologies with the service demand uniformly distributed among cells, i.e., $V_{l} \approx V_{k}, \forall l \neq k$. The service demand in the $l^{\text {th }}$ cell is given by

$$
V_{l}=V \int_{\mathcal{A}_{l}} \delta(\boldsymbol{a}) d \boldsymbol{a} .
$$

Thus, for a given network topology $\mathcal{T}$, the service demand share $\mathcal{V}$ is defined as follows: $\mathcal{V}=\left\{V_{1}, V_{2}, \cdots, V_{L}\right\}$. In general, centroidal Voronoi algorithms do not produce network topologies with uniform service demand share; however, power Voronoi diagrams allow independent calibration of cells by adjusting weights, as in (8). Thus, Algorithm 1 employs both mass centroids and power Voronoi diagrams to obtain network topologies in which the service demand is uniformly distributed among cells, which requires network topologies with different cell sizes.

Algorithm 1 requires as inputs a random set of $L$ points $\mathcal{L}^{\mathrm{R}} \subset \mathcal{A}$, a density function $\delta$, and a set of parameters that controls the operation of the algorithm as described next. Line 3 indicates the execution of $N$ iterations of the standard Centroidal Voronoi Algorithm [10] in order to obtain a good starting point for the loop between Lines 5 and 14. At each iteration, the centroids are recalculated from the previous network topology (Line 5), generators are updated (Line 6), power Voronoi diagrams are computed (Line 7), and the service demand share $\mathcal{V}$ of the new topology is calculated (Line 8). At each iteration, the weight of the cell with the highest demand is updated (Line 10) and the reduction factor $\Delta$ is updated every $T$ iterations to control the stability/convergence of the algorithm (Line 12). The algorithm finishes when the Coefficient of Variation $(\mathrm{CoV})^{2}$ of the service demand share is

\footnotetext{
${ }^{2} \mathrm{The} \mathrm{CoV}$ is a standardized measure of dispersion and it is defined as the ratio of the standard deviation to the mean.
} 


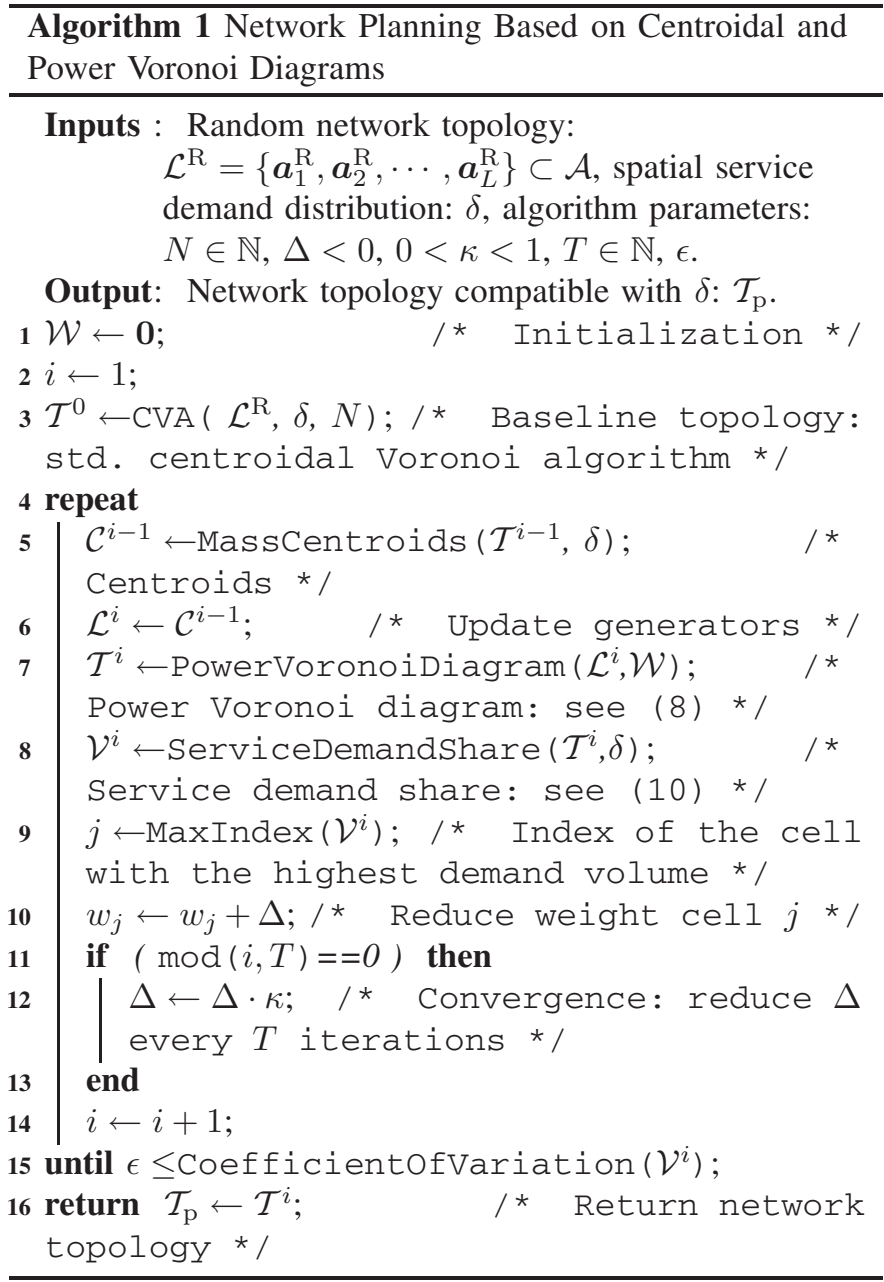

smaller than a threshold $\epsilon$ (Line 15), i.e., the service demand is well distributed among cells. As it will be seen, Algorithm 1 allows the adjustment of network topologies to homogenize the service demand share; and indeed, a simple variation of it (without updating the site locations) could also be used for fixed/existing networks.

\section{Power Optimization}

The need for power optimization is intuitively justified by the fact that, when the spatial service demand is very irregular, access points are concentrated in high demand areas (to increase the spatial frequency reuse); leading to very high levels of interference if power is not adjusted proportionally to cell sizes. In the context of Orthogonal Frequency Division Multiple Access (OFDMA) networks, the effect of interference on the load of each cell is accurately described by the load-coupling model introduced in [12]. As in [5], this model is adopted and briefly described here (for the sake of completeness) as a starting point for the optimization framework that is proposed. The load $\alpha$ of a cell is defined as the fraction of resources that is required, on average, to satisfy the service demand. Thus, following [5], [12], the load $\alpha_{l}$ in the $l^{\text {th }}$ cell is given by

$$
\alpha_{l}=\frac{V R_{\mathrm{min}} \log (2)}{B} \int_{\mathcal{A}_{l}} \frac{\delta_{a}}{\log \left(1+\gamma_{a}(\boldsymbol{\alpha}, \boldsymbol{p})\right)} d a .
$$

Here, the factor $\frac{V R_{\min } \log (2)}{B}$ is a constant, $B$ is the system bandwidth, $R_{\min }$ is the target rate, $\mathcal{A}_{l}$ is the coverage of the $l^{\text {th }}$ access point, and $\delta_{a}$ is the relative service demand in the $a^{\text {th }}$ area element (given by the spatial service demand distribution $(\delta)$ under consideration). The network coverage area is $\mathcal{A}=\bigcup \mathcal{A}_{l}$, with $\mathcal{A}_{i} \cap \mathcal{A}_{j}=\emptyset, \forall i \neq j$. The function $\gamma_{a}(\boldsymbol{\alpha}, \mathbf{p})$ is the SIR in the $a^{\text {th }}$ area element. It is a function of the load in other cells and the power allocation, $\alpha \in \mathbb{R}_{+}^{L}$ and $\mathbf{p} \in \mathbb{R}_{+}^{L}$, respectively. The SIR can be expressed as follows:

$$
\gamma_{a}(\boldsymbol{\alpha}, \mathbf{p})=\frac{p_{\hat{l}} G_{\hat{l}, a}}{\sum_{l=1, l \neq \hat{l}}^{L} p_{l} G_{l, a} \alpha_{l}},
$$

where $\hat{l}$ is the index of the serving access point, $p_{l}$ is the transmit power in the $l^{\text {th }}$ access point, and $G_{l a}$ is the average channel gain between the $a^{\text {th }}$ area element and the $l^{\text {th }}$ access point. Note that interference coming from neighbors is scaled by the corresponding load factors ( $\alpha_{l}$ 's). Thus, the so-called Non-linear Load Coupling Equations (NLCE), $f: \mathbb{R}^{L} \rightarrow \mathbb{R}_{+}^{L}$, can be written as follows [12], [27]:

$$
\boldsymbol{\alpha}=\boldsymbol{f}\left(\boldsymbol{\alpha}, \mathbf{p} ; \delta, \mathbf{G}, V, B, R_{\min }\right),
$$

where $\int_{\mathcal{A}} \delta_{a} d a=1$ and $\mathbf{G} \in \mathbb{R}^{L \times A}$ contains information on the network geometry, i.e., the average channel gain between each area element and access point, and $a=1,2, \cdots, A$. Hereafter, for the sake of clarity, (13) is simply written as:

$$
\alpha=f(\alpha, p) .
$$

As indicated, mathematical properties of (14) including existence and uniqueness of solutions are presented in [12], under the assumption that $\mathbf{p}$ is given. The recent papers [27]-[29] include the power vector $\mathbf{p}$ as an optimization variable to achieve several goals, such as minimization of the sum of the loads $\left(\sum \alpha_{l}\right)$ or minimization of the transmit power (the product $\alpha \cdot \mathbf{p}$ ). Recent improvements in the required algorithmic has also been introduced in [30] and [31]. All these excellent contributions have increased our understanding about load-coupling in OFDMA-based cellular networks and provide useful optimization frameworks.

In [27], for instance, notions such as rate satisfiability and load implementability are developed. Essentially, while for every power allocation $\mathbf{p}$ there is a corresponding load pattern $\alpha$, the converse is not true. The authors present important results regarding the existence and computation of $\mathbf{p}$ for a given load pattern $\boldsymbol{\alpha}$ (as long as $\boldsymbol{\alpha}$ is implementable). To that end, an Iterative Algorithm for Power (IAP) [32] is also presented, and its convergence is shown. It was also proven that $\boldsymbol{\alpha}=\mathbf{1}$ is optimal from the energy efficiency point of view. However, having cells operating at full capacity/load could be not advisable from a practical point of view.

In this work, and as a part of the framework presented herein, a different power optimization formulation is presented and studied. In order to be aligned with [13], where a notion of irregularity is defined in terms of the dispersion of the load vector $\boldsymbol{\alpha}$, the proposed power optimization aims at minimizing the variance of $\boldsymbol{\alpha}$, i.e., $\operatorname{Var}\{\boldsymbol{\alpha}\}$, using $\mathbf{p}$ as optimization variable. This simple, yet interesting, approach has the following important and convenient features:

1) Solving the proposed power optimization results in a uniform load pattern $\bar{\alpha} \cdot \mathbf{1}$, with $\bar{\alpha} \in \mathbb{R}_{+}$. The scalar $\bar{\alpha}$ corresponds to the uniform (common to all cells) 


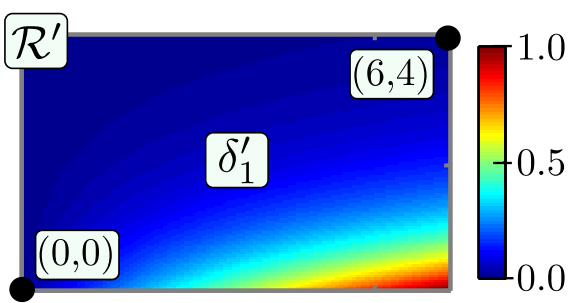

(a)

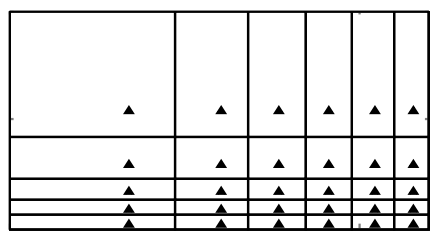

(d)

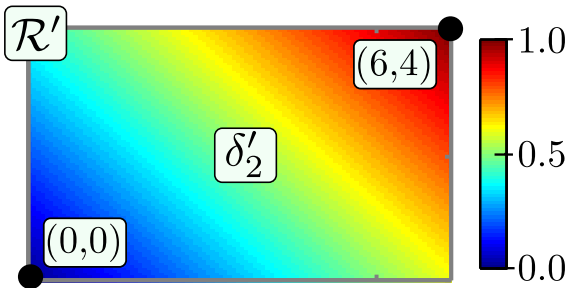

(b)

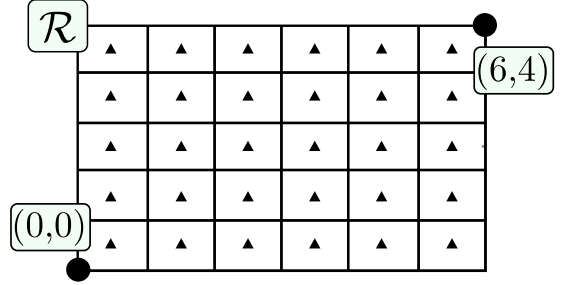

(c)

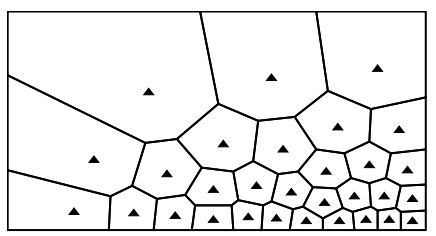

(e)

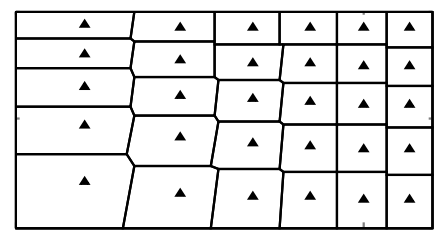

(f)

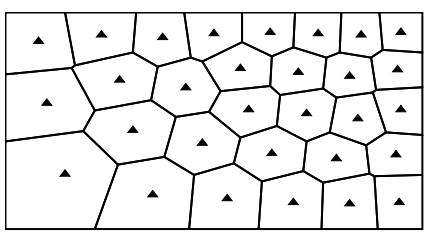

$(\mathrm{g})$

Fig. 5. Examples to illustrate the use of the proposed spatial mappings. (a) $\delta_{1}^{\prime}\left(x^{\prime}, y^{\prime}\right)=x^{\prime} e^{-y^{\prime}}$. (b) $\delta_{2}^{\prime}\left(x^{\prime}, y^{\prime}\right)=x^{\prime}+y^{\prime}$. (c) Synthetic network topology: $\mathcal{T}_{\mathrm{c}}$. (d) Mapping: $\mathcal{T}_{\mathrm{c}} \stackrel{g_{1 \mathrm{~m}}^{-1}}{\longrightarrow} \mathcal{T}_{1 \mathrm{~m}}^{\prime}$. (e) Mapping: $\mathcal{T}_{\mathrm{c}} \stackrel{g_{1 \mathrm{c}}^{-1}}{\longrightarrow} \mathcal{T}_{1 \mathrm{c}}^{\prime}$. (f) Mapping: $\mathcal{T}_{\mathrm{c}} \stackrel{g_{2 \mathrm{~m}}^{-1}}{\longrightarrow} \mathcal{T}_{2 \mathrm{~m}}^{\prime}$. (g) Mapping: $\mathcal{T}_{\mathrm{c}} \stackrel{g_{2 \mathrm{c}}^{-1}}{\longrightarrow} \mathcal{T}_{2 \mathrm{c}}^{\prime}$.

load level obtained when (15) is solved, i.e., once $\mathbf{p}^{\star}$ is applied. This means that cells are equally loaded. As remarked in [2], this is a very important target in planning, where distributing the service demand as evenly as possible is desirable. Indeed, the approach provides a better result because having uniform service demand share does not imply a uniformly loaded network. The proposed optimization does provide the power allocation that is required to achieve the aforementioned important network planning target; and it is done taking into account the load-coupling model (including service demand spatial distribution, interference, and so on). In addition, as it is discussed in [13], achieving the previous goal also maximizes the service demand volume the network is able to manage.

2) No $\alpha$ needs to be specified beforehand. The optimization converges to a resulting uniform load pattern $\bar{\alpha} \cdot \mathbf{1}$, where $\bar{\alpha}$ does not need to be known in advance.

3) The spare capacity is maximized, thus providing robustness as the maximum network-wide protection against instantaneous traffic variations is obtained.

The proposed power optimization can be written as follows:

$$
\begin{aligned}
& \underset{\mathbf{p}}{\operatorname{minimize}} \operatorname{Var}\{\boldsymbol{\alpha}\}, \\
& \text { subject to }: \boldsymbol{\alpha}=\boldsymbol{f}(\boldsymbol{\alpha}, \mathbf{p}), \\
& \mathbf{p} \in \mathbb{R}_{+}^{L} .
\end{aligned}
$$

Thus, by applying $\mathbf{p}^{\star}$, the load of each cell becomes equal to $\bar{\alpha}$. Once $\mathbf{p}^{\star}$ is applied, the network load level, i.e., the value of $\bar{\alpha}$, can be modified by varying the variables $V, B$, and $R_{\min }$. Analogously, any power allocation $\kappa \cdot \mathbf{p}^{\star}$, with $\kappa \in \mathbb{R}_{+}$, is also a solution of (15). In practice, actual levels must consider coverage criteria, as a minimum received power is required. Problem (15) can be addressed by means of solvers based on interior-point methods [33] or through heuristics, such as IAP [27], [32]. Convergence and uniqueness aspects are discussed in Appendix VIII. It is important to point out that solving (15) provides the power allocation for the data channels, while keeping the cells (the regions $\mathcal{A}_{l}$ ) fixed.
This can be regarded as a form of load balancing [34] that, in contrast to existing methods based on cell range adjustments (cell-breathing like schemes), do not transfer traffic from one cell to another, but compensate the load pattern by adjusting interference conditions in the network.

\section{NUMERICAL RESULTS}

In this work, a generalization of the direct mapping $F$ (see Fig. 1) in terms of the composition of two functions has been presented. As it is explained in Section III, and shown in Fig. 2b, the direct mapping $F$ can be written as follows: $F=g \circ f$, where $f$ is a conformal mapping and $g$ is a non-conformal (rectangle-onto-rectangle) mapping. Given that computation and use of the conformal mapping $f$ (and its inverse $f^{-1}$ ) has been fully addressed in [5], the numerical examples illustrate the computation, use, and performance of the new components of the mapping $F$, i.e., the mappings $g$ and $g^{-1}$ (see Figs. 2b and 4) between the domains $\mathcal{R}$ and $\mathcal{R}^{\prime}$, in Sections VII-A and VII-B. Section VII-C presents the results regarding power optimization, and finally, a comparative perspective is provided in Section VII-D.

\section{A. Spatial Mappings}

The setting used in the examples is shown in Fig. 5, where Figs. 5a-5c illustrate the domains $\mathcal{R}^{\prime}$ and $\mathcal{R}$. The spatial service demand distributions $\delta_{1}^{\prime}$ and $\delta_{2}^{\prime}$ are represented in Figs. 5a and 5b, respectively; while the synthetic network topology $\mathcal{T}_{\mathrm{c}}$ is shown in Fig. $5 \mathrm{c}$. The topology $\mathcal{T}_{\mathrm{c}}$ was obtained through the dimensioning analysis in the canonical domain (Section IV). For that, the following assumptions were made:

- Service demand volume: $V=90 \mathrm{~s} / 130 \mathrm{~ms}=692.3$ users (on average); based on the average session time and average inter-arrival time, $90 \mathrm{~s}$ and $130 \mathrm{~ms}$, respectively.

- System bandwidth: $B=20.0 \mathrm{MHz}$.

- Minimum user rate: $R_{\min }=1.0 \mathrm{Mbps}$.

- Average channel gain: $G=d^{-\beta} ; d$ is the distance and $\beta=3$ is the propagation exponent.

- Uniform power allocation: $\mathbf{p}=\mathbf{1}$, and uniform service demand distribution. 

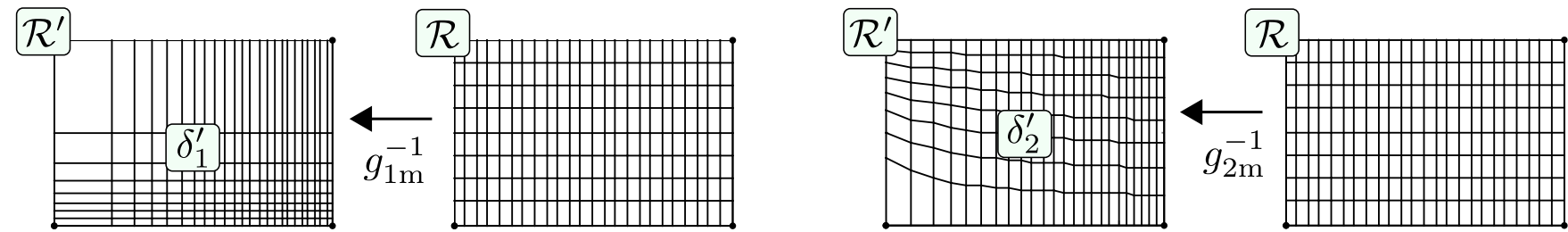

Fig. 6. Illustration of the non-conformal mappings used in the examples.

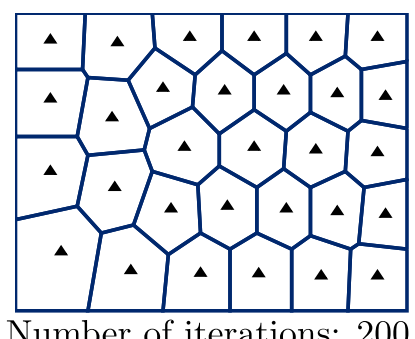

Number of iterations: 200

(a)

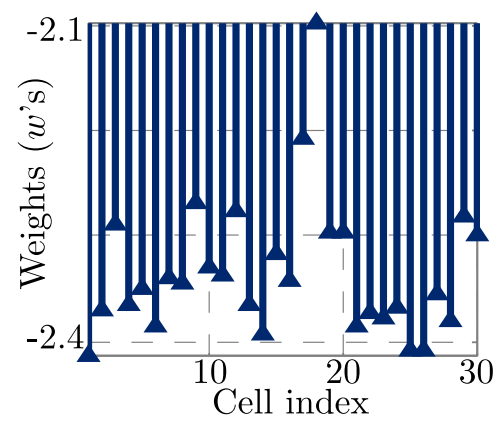

(d)

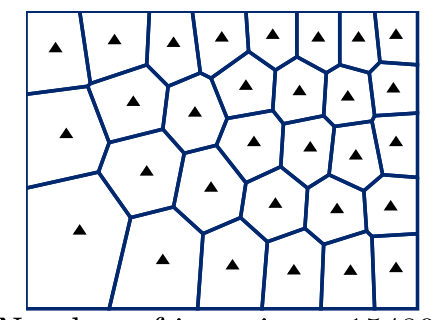

Number of iterations: 15480

(b)

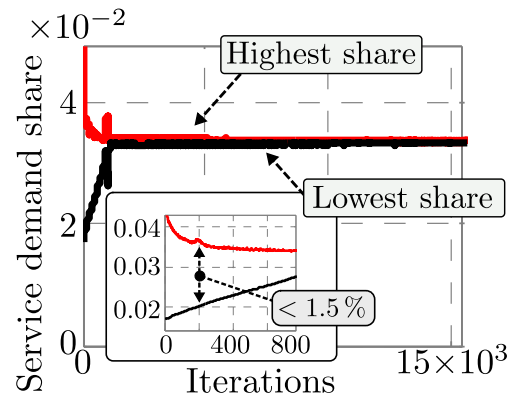

(e)

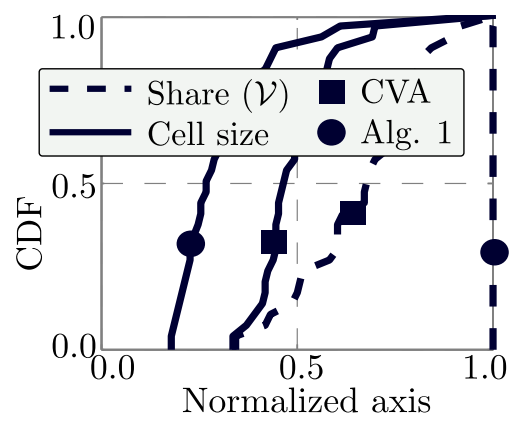

(c)

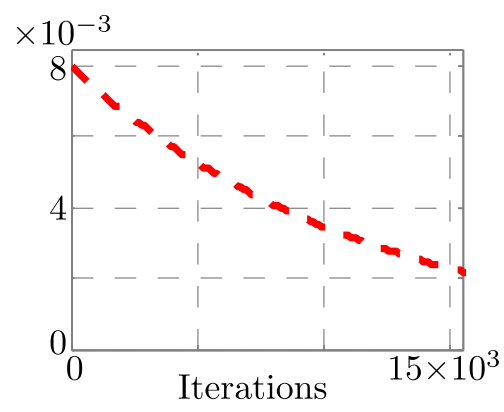

(f)

Fig. 7. Operation of Algorithm 1 with $\delta_{2}^{\prime}$. (a) Topology after standard CVA. (b) Topology after Algorithm 1. (c) Share and cells adjustment. (d) Resulting weights. (e) Load balancing. (f) Reduction of $\Delta$.

A target load was $\bar{\alpha}_{\mathrm{c}} \approx 0.9$ for the canonical domain. Then, a number of base stations $L=30$ was found using a rectangular cell layout as shown in Fig. 5c. Recall that the same parameters are assumed for the domain $\mathcal{R}^{\prime}$, but service demand distribution is obtained according to $\delta_{1}^{\prime}$ and $\delta_{2}^{\prime}$. The different mappings of the topology $\mathcal{T}_{\mathrm{c}}$ onto $\mathcal{R}^{\prime}$ are illustrated in Figs. 5d-5g. The subscripts of the mappings $g^{-1}$ are composed of one number ( 1 or 2$)$ to refer to the spatial service demand distribution $\left(\delta_{1}^{\prime}\right.$ or $\left.\delta_{2}^{\prime}\right)$ that is used to create the mapping, and one letter to indicate the method, i.e., ' $\mathrm{m}$ ' for non-conformal mapping (Section V-A) and ' $\mathrm{c}$ ' for centroidal Voronoi algorithms (Section V-B). For instance, $g_{1 \mathrm{~m}}^{-1}$ (Fig. 5d) indicates the non-conformal mapping of $\mathcal{T}_{\mathrm{c}}$ from $\mathcal{R}$ onto $\mathcal{R}^{\prime}$, for $\delta_{1}^{\prime}$. The same subscripts are used to refer to the nonuniform topologies ( $\mathcal{T}^{\prime}$ 's, Figs. 5d-5g) created in each case.

Note that $\delta_{1}^{\prime}$ (Fig. 5a) corresponds to a case of statistical independence between $x^{\prime}$ and $y^{\prime}$, while $\delta_{2}^{\prime}$ (Fig. 5b) does not. Hence, $g_{1 \mathrm{~m}}^{-1}$ can be expressed as (2) and $g_{2 \mathrm{~m}}^{-1}$ can be expressed as either (5) or (6).

The mapping $g_{1 \mathrm{~m}}^{-1}$ is given by

$$
x^{\prime}=u(x)=\sqrt{6 x},
$$

and

$$
y^{\prime}=v(y)=-\log \left(1-\frac{y}{4}\left(1-e^{-4}\right)\right) .
$$

The mapping $g_{2 \mathrm{~m}}^{-1}$ is given by

$$
x^{\prime}=u(x)=\frac{1}{2}(-4+\sqrt{16+40 x}),
$$

and

$$
y^{\prime}=v(x, y)=-u(x)+\sqrt{[u(x)]^{2}+y[2 u(x)+4]} .
$$

Both $g_{1 \mathrm{~m}}^{-1}$ and $g_{2 \mathrm{~m}}^{-1}$ are illustrated in Fig. 6. It becomes clear how the mappings compress the space (in $\mathcal{R}^{\prime}$ ) where the service demand is high. Thus, we obtain service provision that is compatible with the service demand, i.e., more access points where the demand is concentrated.

Regarding the mappings $g_{1 \mathrm{c}}^{-1}$ and $g_{2 \mathrm{c}}^{-1}$, evidently they cannot be expressed in closed form as resulting topologies are obtained after the execution of Algorithm 1. However, it is recalled that the key step is the calculation of the mass centroids for each cell at each iteration according to (9). This is the mechanism by which Algorithm 1 achieves higher density of access points where the service demand is concentrated. The operation of Algorithm 1 is illustrated in Fig. 7 using the function $\delta_{2}^{\prime}$ as example. Similar operation pattern is obtained for $\delta_{1}^{\prime}$. Figs. 7a corresponds to the network topology obtained after the execution of 200 iterations the standard centroidal Voronoi algorithm (Line 3 in Algorithm 1). In general, centroidal 


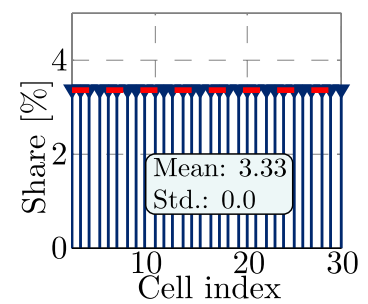

(a)

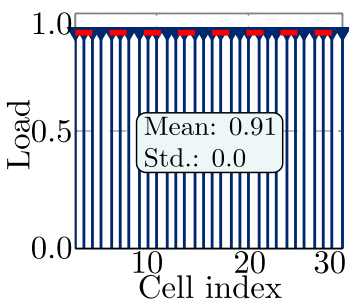

(f)

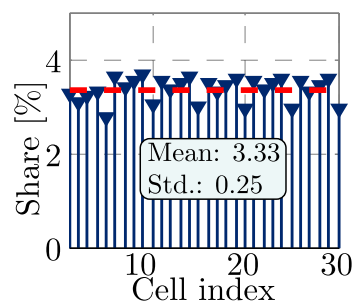

(b)

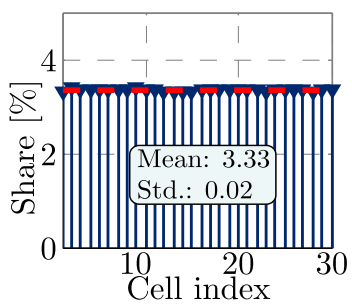

(c)

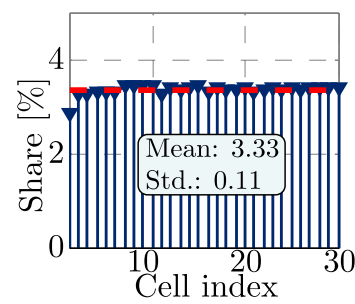

(d)

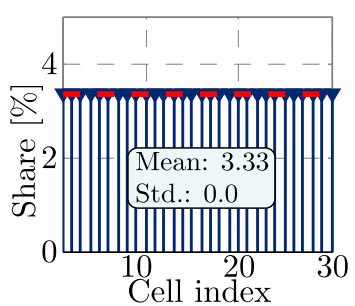

(e)

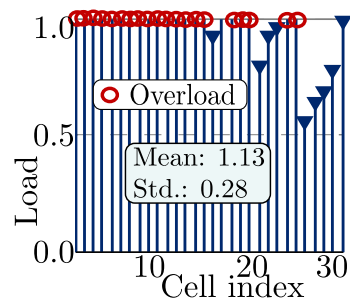

(g)

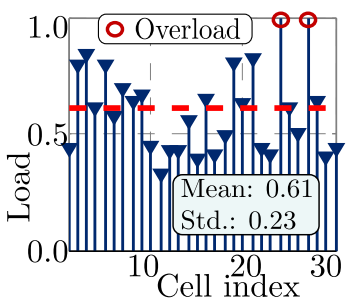

(h)

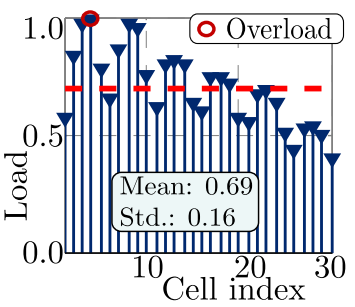

(i)

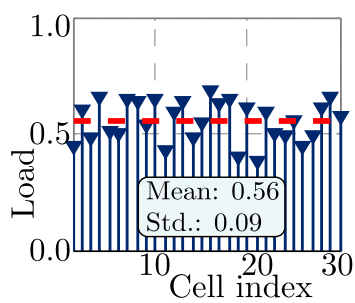

(j)

Fig. 8. Service demand share and load pattern (under uniform power allocation). (a) Share: $\mathcal{T}_{\mathrm{c}}$. (b) Share: $\mathcal{T}_{1 \mathrm{~m}}^{\prime} \cdot(\mathrm{c}) \mathrm{Share:} \mathcal{T}_{1 \mathrm{c}}^{\prime}$. (d) $\operatorname{Share:} \mathcal{T}_{2 \mathrm{~m}}^{\prime}$ (e) Share: $\mathcal{T}_{2 \mathrm{c}}^{\prime}$. (f) Load: $\mathcal{T}_{\mathrm{c}}$. (g) Load: $\mathcal{T}_{1 \mathrm{~m}}^{\prime}$. (h) Load: $\mathcal{T}_{1 \mathrm{c}}^{\prime}$. (i) Load: $\mathcal{T}_{2 \mathrm{~m}}^{\prime}$. (j) Load: $\mathcal{T}_{2 \mathrm{c}}^{\prime}$.

Voronoi algorithms do not result in homogeneous service demand share $\left(V_{l} \approx V_{k}, \forall l \neq k\right)$, however, they provide a good starting point for Algorithm 1. The network topology after the execution of Algorithm 1 is shown in Fig. 7b. Fig. 7c shows a comparative perspective between the standard Centroidal Voronoi Algorithm (CVA) and Algorithm 1, associated in the figure to squares and circles, respectively. Cumulative Distribution Functions (CDFs) of cells size (solid patterns) and service demand share (dash patterns) are shown. All the CDFs are normalized, and hence, $x$-axis also goes from 0 to 1 . CDFs of cells size indicate diversity in terms of cells area size, and CDFs of service demand share indicate how well distributed among cells the service demand is. Clearly, Algorithm 1 succeeds in achieving uniform service demand share, while CVA does not. As explained earlier, Algorithm 1 gradually adjusts the weights of the power Voronoi diagram (Fig. 7d), such that the cell with the highest share reduces its coverage, while cells with less demand tends to increase its coverage. The net result is an increase in cell size range (with respect to CVA), as it is shown in Fig. 7c. Note that the CDF of cells size for the CVA indicates higher cell size homogeneity, which can be verified visually by looking at Figs. 7a and 7b. Fig. 7e shows the evolution of the highest and lowest cell share in the network. In the example, Algorithm 1 reduces in 200 iterations the gap between the cells with highest and smallest share in less than $1.5 \%$. The figure illustrates the asymptotic convergence of Algorithm 1. As mentioned, the reduction factor $\Delta$ (see Algorithm 1) is gradually decreased to enhance the convergence of the algorithm as shown in Fig. 7f.

\section{B. Service Demand Share and Load Patterns}

The service demand share and load patterns of the topologies illustrated in Figs. 5c-5g are shown in Fig. 8. The service demand share and load pattern of the canonical domain (Fig. 5c) are shown in Figs. 8a and 8f, respectively. As indicated earlier, the conditions of the canonical domain (uniform spatial service demand distribution, uniform service demand share, and same amount of received interference per cell), result in a flat load pattern, i.e., $\alpha_{l}=\alpha_{k}, \forall l \neq k$. In the example, each one of the 30 cells has a share of $3.33 \%$ of the service demand, which always results in a load equal to 0.91 .

The service demand share of the topologies $\mathcal{T}_{1 \mathrm{~m}}^{\prime}$ and $\mathcal{T}_{2 \mathrm{~m}}^{\prime}$ created by the non-conformal mapping are shown in Figs. 8b and 8d, respectively. In these cases, there is a small unbalance in the service demand share due to the fact that the mappings are only used to map the access points (and not the boundaries) from $\mathcal{R}$ to $\mathcal{R}^{\prime}$. Once in $\mathcal{R}^{\prime}$, cells are defined using standard Voronoi diagrams, i.e., each point is associated to its closest access point. ${ }^{3} \mathrm{~A}$ perfect service demand share could be attained, for instance, by using Algorithm 1 to adjust the cells sizes of these topologies; however, they are intentionally kept in this manner to show that the power optimization proposed in Section VI does not require uniform service demand share. Their resulting load patterns, when uniform power allocation (cells transmit in data channels with the same power) is assumed, are shown in Figs. 8g and 8i. Note that, as expected, uniform power allocation is not a good idea for topologies with very different access points densities, such as $\mathcal{T}_{1 \mathrm{~m}}$, due to the high interference that is created. In Fig. 8g, most of the cells get load factors greater than one (which in practice means outage), indicated by red circles. In Fig. 8i only one cell has load larger than one, but still the load pattern is very irregular, i.e., the network is far from being uniformly loaded.

The topologies obtained using Algorithm 1 feature uniform service demand share as it can be seen from Figs. 8c and 8e. The corresponding load patterns (under uniform power allocation) are shown in Figs. 8h and 8j. In the light of these examples, it becomes clear that the more irregular the topology is, the less feasible the uniform power allocation assumption, and hence, the larger the need for power optimization. However, the average network load obviously depend on the service demand volume $(V)$, but it is important to recall that this dependency is highly non-linear. Note that, while in case of $\mathcal{T}_{1 \mathrm{c}}$ (Fig. 8h), there are two cells in outage, in case of $\mathcal{T}_{2 \mathrm{c}}$ (Fig. 8j), the average cell load is around 0.6, with no outage. Evidently, the assumption of uniform power allocation is much less valid

\footnotetext{
${ }^{3}$ Two additional methods, along with their pros and cons, are explained in $[5, \mathrm{Sec} . \mathrm{V}-\mathrm{C}]$.
} 


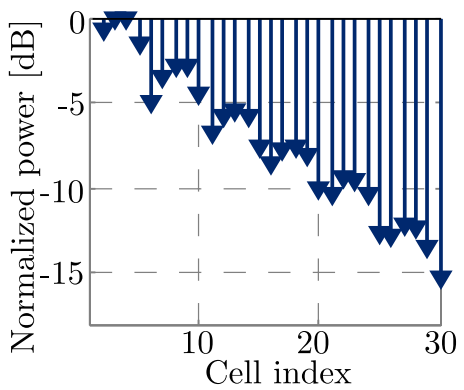

(a)

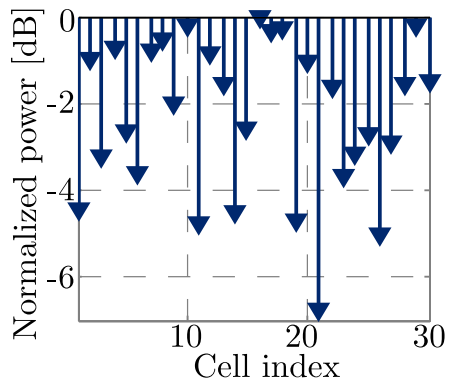

(d)

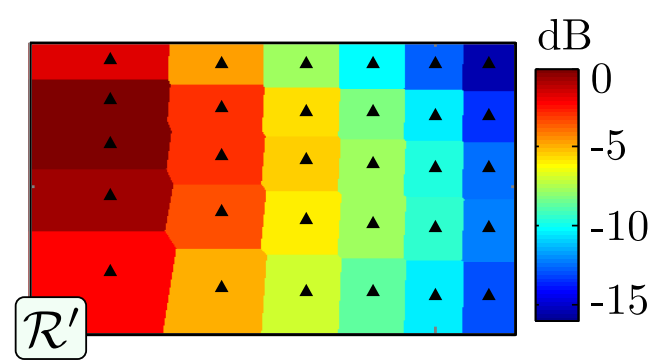

(b)

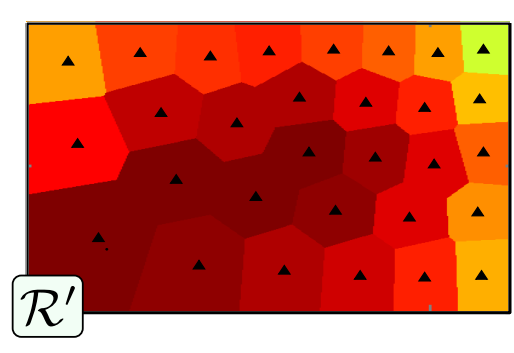

(e)

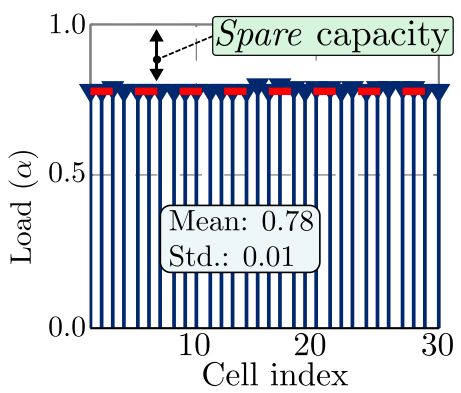

(c)

Fig. 9. Use of power optimization to obtain uniformly loaded networks with the highest spare capacity. (a) Optimized power for $\mathcal{T}_{2} \mathrm{~m}$. (b) Spatial power allocation pattern for $\mathcal{T}_{2 \mathrm{~m}}$. (c) Uniform load pattern. (d) Optimized power for $\mathcal{T}_{2 \mathrm{c}}$. (e) Spatial power allocation pattern for $\mathcal{T}_{2 \mathrm{c}}$. (f) Uniform load pattern.

for $\mathcal{T}_{1 \mathrm{c}}$ than for $\mathcal{T}_{2 \mathrm{c}}$, as the former is much more irregular than the latter, see Figs. 5e and 5g. The following examples illustrates the use of power optimization.

\section{Load Balancing Through Power Optimization}

As indicated before, optimizing the power allocated to the data channels is a convenient alternative to achieve load balancing, without need for transferring service demand from one cell to another; for instance, by adjusting the power of cellspecific reference signals [26] that are used for cell-selection. In order to illustrate the use of power optimization, the topologies $\mathcal{T}_{2 \mathrm{~m}}$ and $\mathcal{T}_{2 \mathrm{c}}$ (Figs. 5f and $5 \mathrm{~g}$ ), produced in $\mathcal{R}^{\prime}$ by the mappings $g_{2 \mathrm{~m}}^{-1}$ and $g_{2 \mathrm{c}}^{-1}$, respectively, and the non-uniform spatial service demand distribution $\delta_{2}^{\prime}$ are considered. This does not imply any loss of generality with respect to its use in the physical domain $\mathcal{A}$, as the power optimization proposed in Section VI is topology and domain agnostic. Figs. 9a and 9d show the optimized power vector $\mathbf{p}^{\star}$ in both cases. A visual representation is also provided in Figs. $9 \mathrm{~b}$ and $9 \mathrm{e}$, where the power allocated to each cell is normalized (with respect to the highest value) and expressed in $\mathrm{dB}$. Both figures have the same scale: from $0 \mathrm{~dB}$ (the highest power) to $-16 \mathrm{~dB}$. It can be observed that there is a certain correlation between the power allocated to each cell and its size, i.e., smaller cells tend to be allocated with less power. However, this is not a rigid rule as shown in the figures, but a trend that is just intuitively expected. The actual optimal power allocation depends on the spatial service demand distribution and network topology/geometry (site locations and cells). The resulting uniform load patterns are shown in Figs. 9c and 9f, where it is evident that the proposed power optimization succeeds in finding a power allocation able to homogenize the load pattern in both cases. Thus, finding the solution of (15) not only leads to a power allocation in which the cells are uniformly loaded (a nice feature from network planning perspective), but also maximizes the spare capacity in the network. Indeed, the resulting uniform load level $\bar{\alpha}$ is the minimum possible for a given $V, R_{\text {min }}$, and $B$. In Appendix VIII, uniqueness aspects are discussed. This is important because spare capacity, indicated in Figs. 9c and 9f, provides a natural protection against instantaneous service demand variations that would appear in real deployments. ${ }^{4}$ If we look at these results from a comparative point of view, taking the network topology $\mathcal{T}_{2}$ as reference, we can observe not only the performance difference between the two schemes (mapping and iterative algorithm), but also the remarkable performance gap with respect to the original scheme proposed in [5] that lacks of power optimization. Focusing on Figs. $8 \mathrm{i}$ and $9 \mathrm{c}$, it is clear that in the former case (Fig. 8i) the network is quite unbalanced in terms of cell load with cells in outage and several others about to be in outage if minor service demand variations occur. In the later case (Fig. 9c), the load of the cells is 0.79 , meaning that every cell has a spare capacity of $21 \%$, on average. A similar analysis holds between Figs. 8j and 9f, although in this case, the gain is only in terms of homogeneity since the power optimization achieves uniform cell load and the service demand volume considered is relatively low. Based on this analysis, it can be concluded that power optimization is a substantial enhancement from the practical point of view.

\section{Final Remarks}

All in all, the results have shown the effectiveness and usability of both spatial mappings and centroidal-based algorithms with power Voronoi diagrams (Algorithm 1). As in many other instances, different approaches have advantages and drawbacks, and in this context the word 'better' is not really suitable. The use of one or another depends on preferences, requirements, available resources, and the

\footnotetext{
${ }^{4}$ Recall that load values calculated using (11) are average figures.
} 
characteristics of the particular problem to be addressed. A short list of practical rules-of-thumbs are provided next:

$\checkmark$ Scale of the Problem: Based on our experience, if the problem requires the deployment of a large number of cells (e.g., $L \in[75,1000]$ ), operating with spatial mappings is most like a good choice, because the execution of centroidal Voronoi algorithms could be very expensive, as centroids need to be computed for each cell at each iteration. In these cases, the compactness of spatial mappings is really a desirable feature, as it was shown in the examples provided in [5]. For relatively small deployments $(L<75)$, obtaining perfect service demand share is a nice plus that can be obtained by means of Algorithm 1. However, as it was shown, uniform service demand share is not a requirement for power optimization.

$\checkmark$ Context Variables/Assumptions: The spatial service demand distributions used herein have been selected to be functions leading to closed-form mappings and centroid solutions. They also exemplify statistical independence and non-statistical independence. As indicated in [5], conformal mapping as well as the mappings introduced herein not only admit, but require numerical solution in most of the practical problems. Examples allowing analytic solutions, such as $\delta_{1}^{\prime}$ and $\delta_{2}^{\prime}$, are essentially reserved for theoretic/academic purposes. Therefore, more complex functions used to approximate the spatial service demand distribution would require numerical evaluation, same as spatial distributions completely given in numerical terms. The particular structure of the $\delta$ that is assumed could be more suitable for one approach or another. It is also important to look at the definition of the physical domain. While conformal mapping provides a general setting for mapping arbitrary polygons onto rectangles, the assumption of defining $\mathcal{A}$ as a rectangle would be valid in many practical contexts as well.

$\checkmark$ Network Fine-Tuning: The proposed framework is a tool for planning and optimization purposes; and it is a complementary approach to existing methods, such as system level simulations or stochastic geometry. Hence, it is perfectly valid to fine-tune the resulting/obtained network topologies using the methods proposed herein, i.e., adjusting cells coverage by means of power Voronoi diagrams or power optimization for load balancing as proposed in Section VI; or to resort to other alternatives, such as the power optimization for energy efficiency proposed in [27] or existing load balancing methods [34]. Planning and radio access optimization is a difficult problem, and hence, there is not a unique recipe. The framework presented herein provides additional effective tools to aid at these tasks.

$\checkmark$ Intercell Interference Coordination and Signaling: It is worth noting that changes in the interference statistics due to use of e.g. Intercell Interference Coordination (ICIC) may have some impact on the optimal network deployment especially when service demand distribution is irregular and very dense in some locations. ICIC could be modelled through channel gains in equation (12). In this work, such modelling has not been considered since it could unnecessarily move the focus from network planning to interference coordination. Accordingly, signalling related to ICIC is also out of the scope of this paper.
It is good to acknowledge that the presented deterministic network planning concept is primarily developed to serve as a tool for analytic investigations with aim to make dependencies between different parameters/factors visible. We note that similar planning result can be obtained using good heuristic methods. Furthermore, the current computational efficiency of the first algorithmic realizations of the methodology is not very high (it takes some tens of minutes to run algorithms in a baseline laptop). Yet, the computation time is not a bottleneck in traditional network planning that is made offline. In future networks with moving small cells (e.g., relays in buses) network planning may become a more dynamic task and computation time becomes a more important factor. Finally, the computational burden heavily depends on the size of the network and the service demand 'irregularity' that reflects also to the properties of the spatial mappings. It is a future research topic to design principles for the network partition to allow parallel and more effective algorithm realizations.

\section{CONCLUSIONS AND RESEARCH DIRECTIONS}

Planning and optimization are tasks that certainly need to go hand-in-hand to maximize the profit and performance of current and future cellular systems. In this work, key contributions to the planning and optimization framework based on canonical domains and spatial mappings, originally introduced in [5], have been presented. These novelties include more general and versatile mappings, new algorithmic tools, and power optimization schemes. The results confirm the potential and promising research perspectives of the proposed framework, in which having an statistical description of the spatial service demand distribution, is of utmost importance. As it was explained, planning is a very tough problem, and to address it in the context of future $5 \mathrm{G}$ systems, several tools need to be combined to achieve the expected outcomes. In this sense, our framework is complementary to well-known existing methods, such as system level simulations or stochastic geometry, each of which has advantages and drawbacks. However, the proposed framework provides not only another methodology (with pros and cons as well), but also a new angle to look at this problem. Because this idea is in its infancy, the authors are confident that many enhancements are to come and this contribution will benefit both academy and industry.

Our current research efforts are aligned in the following directions:

1) Indoor network planning. The goal is to evolve the proposed framework for planning and optimization of indoor deployments, using cutting-edge mapping techniques developed by the authors, such as [35].

2) Service demand in 3 dimensions. Provide means to use the current art to study realistic cases where the service demand is given in 3 dimensions, e.g., to include buildings.

3) HetNets: Heterogeneous networks is another direction to be addressed. Methods based on the idea of composition and separation of tiers are currently under study. One clear application of this is to consider cases where network already exist, but some capacity enhancements are pursuit by means of additional access points, i.e., optimal Radio Access Network expansion. Also, integrating more general metrics to account with sectorization is another clear extension of this work.

4) Uplink: To explicitly take into account within the optimization framework the particularities of both downlink 


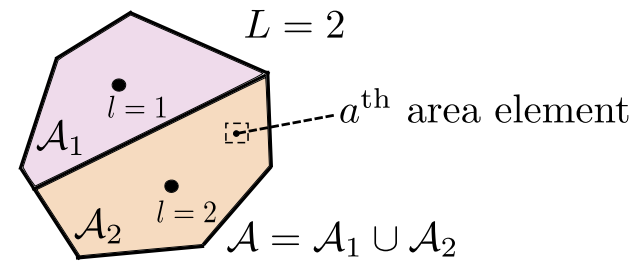

Fig. 10. Toy example: small cellular network composed of two cells.

and uplink is a natural research question to be considered within the evolution of the proposed scheme.

5) Unmanned Aid Vehicles (UAVs): Due to its nature, the proposed framework is highly suitable to be used in the positioning of UAVs, where mobility can also be assumed for the access points. The temporal evolution of the service demand is another clear path to go.

\section{APPENDIX}

\section{POWER Optimization: CONVERGENCE AND UniquenEsS}

In this appendix, convergence and uniqueness aspects of the following minimization problem are discussed:

$$
\begin{aligned}
\underset{p}{\operatorname{minimize}} \operatorname{Var}\{\boldsymbol{\alpha}\}, \\
\text { subject to : } \boldsymbol{\alpha}=\boldsymbol{f}(\boldsymbol{\alpha} ; \mathbf{p}) \\
\mathbf{p} \in \mathbb{R}_{+}^{L},
\end{aligned}
$$

where $\boldsymbol{\alpha} \in \mathbb{R}_{+}, \mathbf{p} \in \mathbb{R}_{+}$, and the $l^{\text {th }}$ element of $\boldsymbol{\alpha}, \alpha_{l}$, is given by

$$
\alpha_{l}=f_{l}(\boldsymbol{\alpha}, \mathbf{p})=K \sum_{a \in \mathcal{A}_{l}} \frac{\delta_{a}}{\log \left(1+\frac{p_{l} G_{l, a}}{\sum_{i=1, i \neq l}^{L} p_{i} G_{i, a} \alpha_{i}}\right)},
$$

with $\sum_{l=1}^{L} \sum_{a \in \mathcal{A}_{l}} \delta_{a}=1, K \in \mathbb{R}_{+}$, and $G_{l, a} \in$ $[0,1) \forall\left(l \in\{1,2, \cdots, L\} \wedge a \in \mathcal{A}=\bigcup_{l=1}^{L} \mathcal{A}_{l}\right)$. The previous problem is a discrete version of (15), where the coverage region of each access point (i.e., the sets $\mathcal{A}_{l}$ 's) is divided into many small area elements. For the sake of clarity, and without loss of generality, a basic network composed of two cells $(L=2)$, as shown in Fig. 10, is considered.

\section{A. Guaranteed Convergence of Interior Point Methods}

The problem (20) is quadratic. The gradients of the constraint functions $\alpha_{l}-f_{l}(\boldsymbol{\alpha}, \mathbf{p})=0$ and $p_{l}>0$ are linearly independent, since by construction the matrix of gradients is diagonally dominant. Notice, that the diagonal elements are identically $=1$. Thus, the convergence of interior point methods is guaranteed.

\section{B. Remark on Uniqueness of Minimal Load Vector}

Let us next focus on the discrete formulation of the simple configuration given above:

$$
\begin{aligned}
& \alpha_{1}=f_{1}(\boldsymbol{\alpha}, \mathbf{p})=K \sum_{a \in \mathcal{A}_{1}} \frac{\delta_{a}}{\log \left(1+\frac{1}{\alpha_{2}} \frac{p_{1} G_{1 a}}{p_{2} G_{2 a}}\right)}, \\
& \alpha_{2}=f_{2}(\boldsymbol{\alpha}, \mathbf{p})=K \sum_{a \in \mathcal{A}_{2}} \frac{\delta_{a}}{\log \left(1+\frac{1}{\alpha_{1}} \frac{p_{2} G_{2 a}}{p_{1} G_{1 a}}\right)} .
\end{aligned}
$$

Let us assume equilibrium and set $\bar{\alpha}=\alpha_{1}=\alpha_{2}$ with naturally $\bar{\alpha} \in \mathbb{R}_{+}$. Next we consider a scaling $\bar{\alpha} \rightarrow c \bar{\alpha}$, where $1 / \bar{\alpha}>c>0$ is a constant, in order to investigate whether for any value $c<1$ there exists a power vector $\hat{\mathbf{p}}$ which also minimizes the problem (20). Inserting the scaling into equations we get

$$
\begin{aligned}
c \bar{\alpha} & =K \sum_{a \in \mathcal{A}_{1}} \frac{c \delta_{a}}{\log \left(1+\frac{1}{\bar{\alpha}} \frac{p_{1} G_{1 a}}{p_{2} G_{2 a}}\right)}, \\
c \bar{\alpha} & =K \sum_{a \in \mathcal{A}_{2}} \frac{\delta_{a}}{\log \left(1+\frac{1}{c \bar{\alpha}} \frac{p_{2} G_{2 a}}{p_{1} G_{1 a}}\right)} .
\end{aligned}
$$

Setting $p_{1}$ and $p_{2}$ to values obtained in the non-scaled minimization in (24) but rewriting (25) as

$$
c \bar{\alpha}=K \sum_{a \in \mathcal{A}_{2}} \frac{\delta_{a}}{\log \left(1+\frac{1}{c \bar{\alpha}} \frac{\hat{p}_{2} G_{2 a}}{\hat{p}_{1} G_{1 a}}\right)},
$$

we can search for a solution (minimizer) to an equation $(24)=(26)$, with $c, \hat{p}_{1}$, and $\hat{p}_{2}$ as free parameters. This equation has only one fixed point with $c=1$ and $p_{1} / p_{2}=$ $\hat{p}_{1} / \hat{p}_{2}$ and we conclude that the obtained $\bar{\alpha}$ is optimal and unique up to scaling of the components of $\mathbf{p}$.

\section{REFERENCES}

[1] M. Jaber, Z. Dawy, N. Akl, and E. Yaacoub, "Tutorial on LTE/LTEA cellular network dimensioning using iterative statistical analysis," IEEE Commun. Surveys Tuts., vol. 18, no. 2, pp. 1355-1383, 2nd Quart., 2016.

[2] S. Wang and C. Ran, "Rethinking cellular network planning and optimization," IEEE Wireless Commun., vol. 23, no. 2, pp. 118-125, Apr. 2016.

[3] F.-H. Tseng, L.-D. Chou, H.-C. Chao, and J. Wang, "Ultra-dense small cell planning using cognitive radio network toward 5G," IEEE Wireless Commun., vol. 22, no. 6, pp. 76-83, Dec. 2015.

[4] E. Oh, B. Krishnamachari, X. Liu, and Z. Niu, "Toward dynamic energyefficient operation of cellular network infrastructure," IEEE Commun. Mag., vol. 49, no. 6, pp. 56-61, Jun. 2011.

[5] D. González G. and J. Hämäläinen, "Looking at cellular networks through canonical domains and conformal mapping," IEEE Trans. Wireless Commun., vol. 15, no. 5, pp. 3703-3717, May 2016.

[6] N. Papamichael and N. Stylianopoulos, Numerical Conformal Mapping: Domain Decomposition and the Mapping of Quadrilaterals. Singapore: World Scientific, 2010.

[7] L. V. Ahlfors, Complex Analysis: An Introduction to the Theory of Analytic Functions of One Complex Variable. New York, NY, USA: McGraw-Hill, 1953.

[8] G. J. Foschini, "Layered space-time architecture for wireless communication in a fading environment when using multi-element antennas," Bell Labs Tech. J., vol. 1, no. 2, pp. 41-59, 1996.

[9] J. H. Shapiro, Composition Operators and Classical Function Theory. New York, NY, USA: Springer-Verlag, 1993.

[10] Q. Du, V. Faber, and M. Gunzburger, "Centroidal Voronoi tessellations: Applications and algorithms," SIAM Rev., vol. 41, no. 4, pp. 637-676, 1999.

[11] Y. Tan, L. Li, and Y. Wang, Dynamic Construction of Power Voronoi Diagram. Berlin, Germany: Springer, 2011, pp. 660-667.

[12] I. Siomina and D. Yuan, "Analysis of cell load coupling for LTE network planning and optimization," IEEE Trans. Wireless Commun., vol. 11, no. 6, pp. 2287-2297, Jun. 2012.

[13] D. González G. and J. Hämäläinen, "Topology and irregularity in cellular networks," in Proc. IEEE Wireless Commun. Netw. Conf. (WCNC), Mar. 2015, pp. 1719-1724.

[14] X. Zhou, Z. Zhao, R. Li, Y. Zhou, and H. Zhang, "The predictability of cellular networks traffic," in Proc. Int. Symp. Commun. Inf. Technol. (ISCIT), Oct. 2012, pp. 973-978.

[15] D. Lee, S. Zhou, X. Zhong, Z. Niu, X. Zhou, and H. Zhang, "Spatial modeling of the traffic density in cellular networks," IEEE Wireless Commun., vol. 21, no. 1, pp. 80-88, Feb. 2014. 
[16] M. Malmirchegini and Y. Mostofi, "On the spatial predictability of communication channels," IEEE Trans. Wireless Commun., vol. 11, no. 3, pp. 964-978, Mar. 2012.

[17] P. J. Olver. (2015). Complex Analysis and Conformal Mapping. [Online]. Available: http://www.math.umn.edu/\%20olver/ln/cml.pdf

[18] L. H. Howell and L. N. Trefethen, "A modified Schwarz-Christoffel transformation for elongated regions," SIAM J. Sci. Statist. Comput., vol. 11, no. 5, pp. 928-949, 1990.

[19] T. A. Driscoll and L. N. Trefethen, Schwarz-Christoffel Mapping, vol. 8. Cambridge, U.K.: Cambridge Univ. Press, 2002.

[20] J. M. Sullivan, "Conformal tiling on a torus," in Proc. Bridges, Coimbra, 2011, pp. 593-596.

[21] D. González G. and J. Hämäläinen, "Planning and optimization of cellular networks through centroidal Voronoi tessellations," in Proc. IEEE 82nd Veh. Technol. Conf. (VTC), Sep. 2015, pp. 1-2.

[22] R. Johnson, I. Miller, and J. Freund, Miller \& Freund's Probability and Statistics for Engineers. Englewood Cliffs, NJ, USA: Prentice-Hall, 2011.

[23] A. Landström, H. Jonsson, and A. Simonsson, "Voronoi-based ISD and site density characteristics for mobile networks," in Proc. IEEE 76th Veh. Technol. Conf. (VTC Fall), Sep. 2012, pp. 1-5.

[24] X. Ge, B. Yang, J. Ye, G. Mao, and Q. Li, "Performance analysis of Poisson-Voronoi tessellated random cellular networks using Markov chains," in Proc. IEEE Global Commun. Conf. (GLOBECOM), Dec. 2014, pp. 4635-4640.

[25] R. Ullah et al., "Voronoi cell geometry based dynamic fractional frequency reuse for OFDMA cellular networks," in Proc. IEEE Int. Conf. Signal Image Process. Appl. (ICSIPA), Oct. 2013, pp. $435-440$.

[26] E. Dahlman, S. Parkvall, J. Sköld, and P. Beming, $3 G$ Evolution: HSPA and LTE for Mobile Broadband, 2nd ed. Oxford, MA, USA: Academic, 2008.

[27] C. K. Ho, D. Yuan, L. Lei, and S. Sun, "Power and load coupling in cellular networks for energy optimization," IEEE Trans. Wireless Commun., vol. 14, no. 1, pp. 509-519, Jan. 2015.

[28] Z.-H. Yang, Y.-J. Pan, M. Chen, H. Xu, and J.-F. Shi, "Cell load coupling with power control for LTE network planning," in Proc. Int. Conf. Wireless Commun. Signal Process. (WCSP), Oct. 2015, pp. 1-5.

[29] L. You, L. Lei, and D. Yuan, "Range assignment for power optimization in load-coupled heterogeneous networks," in Proc. IEEE Int. Conf. Commun. Syst. (ICCS), Nov. 2014, pp. 132-136.

[30] R. L. G. Cavalcante, E. Pollakis, and S. Stańczak, "Power estimation in LTE systems with the general framework of standard interference mappings," in Proc. IEEE Global Conf. Signal Inf. Process. (GlobalSIP), Dec. 2014, pp. 818-822.

[31] R. L. G. Cavalcante, Y. Shen, and S. Stańczak, "Elementary properties of positive concave mappings with applications to network planning and optimization," IEEE Trans. Signal Process., vol. 64, no. 7, pp. 1774-1783, Apr. 2016.

[32] R. D. Yates, "A framework for uplink power control in cellular radio systems," IEEE J. Sel. Areas Commun., vol. 13, no. 7, pp. 1341-1347, Sep. 1995.

[33] F. A. Potra and S. J. Wright, "Interior-point methods," J. Comput. Appl. Math., vol. 124, nos. 1-2, pp. 281-302, 2000.

[34] J. Andrews, S. Singh, Q. Ye, X. Lin, and H. Dhillon, "An overview of load balancing in HetNets: Old myths and open problems," IEEE Wireless. Commun., vol. 21, no. 2, pp. 18-25, Apr. 2014.

[35] H. Hakula, T. Quach, and A. Rasila, "Conjugate function method for numerical conformal mappings," J. Comput. Appl. Math., vol. 237, no. 1, pp. 340-353, Jan. 2013

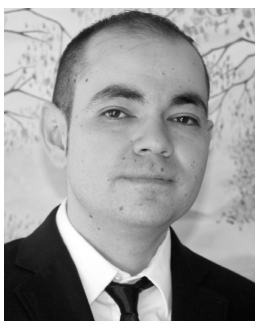

David González G. was born in Chitré, Panama. He received the B.S. degree in electronics and communications engineering from the Universidad de Panamá in 2002, and the master's and Ph.D. degrees from the Universitat Politècnica de Catalunya, Spain, in 2008 and 2013, respectively. From 2002 to 2005, he was a Telecommunication Engineer with Cable Onda, a cable TV operator in Panamá, where he was involved in the integration of digital telephony services. During this period, he also served as a Lecturer with the Universidad Tecnológica de Panamá. In 2014, he joined the Department of Communications and Networking, Aalto University, Finland. His current research activities are focused on the diverse aspects of cellular networks and wireless communications, including interference modeling/coordination, radio access modeling/optimization, and $5 \mathrm{G}$ standardization.

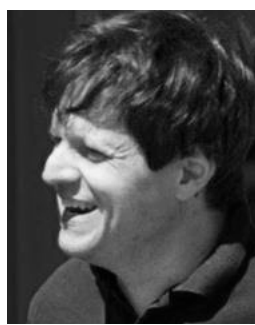

Harri Hakula received the Ph.D. degree in mathematics from the Aalto University School of Science and Technology (formerly known as Helsinki University of Technology), Espoo, Finland, in 1997. He is currently a Senior University Lecturer with the Department of Mathematics and Systems Analysis, Aalto University School of Science and Technology. His research interests include the numerical solution of partial differential equations (PDEs), including stochastic PDEs and numerical conformal mappings.

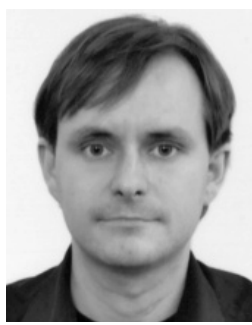

Antti Rasila received the Ph.D. degree in 2005 from the University of Helsinki. He is a Mathematician with Aalto University, Finland. He is also a Docent with the University of Vaasa, Finland, and a Guest Professor with Hengyang Normal University, Hunan, China. In addition, he was involved in computeraided methods in mathematics education, where he has led several international as well as national level research and development projects. He is also interested in the applications of mathematics, computers, and programming. His fields of expertise include conformal invariants, harmonic, and quasiconformal mappings, numerical conformal mappings, and elliptic partial differential equations.

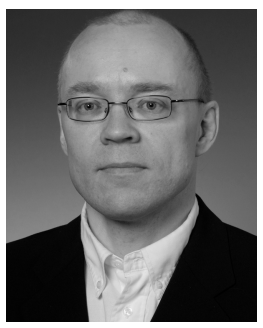

Jyri Hämäläinen received the M.Sc. and Ph.D. degrees from the University of Oulu, Finland, in 1992 and 1998, respectively. From 1999 to 2007, he was a Senior Specialist and Program Manager with Nokia, where he was involved in the various aspects of mobile communication systems and 3GPP standardization. Since 2008, he has been a Professor with the School of Electrical Engineering, Aalto University, where he is currently serving as the Dean. From 2012 to 2014, he was also serving in the management team of Ericsson Research and Development Unit, Oulu. He is author or co-author of 190 scientific publications and 36 U.S. patents or patent applications. His research interests cover mobile and wireless systems, special focus being in dynamic and heterogeneous networks, including, e.g., small cells, multi-antenna transmission and reception techniques, scheduling, and relays. 